\title{
Liste des parasites par ordre systématique
}

Dans cette liste, ne sont pas mentionnés les parasites d'animaux n'appartenant pas à la faune régionale, apportés à la Station de Richelieu pour des recherches expérimentales.

\section{PROTOZOAIRES PARASITES}

\section{Amœbida}

Entamoeba invadens Rodhain 1934

\section{Trypanosomida}

Trypanosomes indéterminés

$$
\begin{aligned}
& \text { id. } \\
& \text { id. } \\
& \text { id. }
\end{aligned}
$$

Trypanosoma cuniculi R. Blanchard $1904=T$. nabiasi
A. Railliet 1895

Trypanosomes indéterminés

id.
id.

Trypanosoma sanguinis

Gruby 1843

Trypanosomes indéterminés

Trypanoplasmes indéterminés id.

\section{HôTES}

Vipera aspis L., expérimentalement

$\begin{array}{cl}\text { sang } & \text { Talpa europaea L. } \\ \text { sang } & \begin{array}{c}\text { Myotis emarginatus (Et. Geof- } \\ \text { froy-St-Hilaire) }\end{array} \\ \text { sang } & \text { Myotis myotis (Borkhausen) } \\ \text { sang } & \text { Plecotus auritus (L.) } \\ \text { id. } & \text { Oryctolagus cuniculus (L.) ferus }\end{array}$

id. Coloeus monedula turrium (Brehm)

id. Garrulus glandarius L.

id. Athene noctua (Scopoli)

id. Falco subbuteo $\mathrm{L}$.

td. Rana esculenta L.
id. $\quad$ Tinca tinca (L.)
td. Gardonus rutilus (L.)
id. Tinca tinca (L.) 


\section{Trichomonadina}

Trichomonas indéterm.

id.

Eutrichomastix sp.

\section{Diplomonadina}

Giardia sp.

id.

Hexamita sp. intestin Arvicola sapidus tenebricus Gerrit Miller

id. Microtus arvalis (Pallas)

id. Vipera aspis L.

intestin Arvicola sapidus tenebricus Gerrit Miller

id. Microtus arvalis (Pallas)

id. Microtus arvalis (Pallas)

\section{Cnidosporidia}

Myxosporidies non identifiées

$$
\text { id. }
$$$$
\text { id. }
$$

id.

Myxobolus sp.

Thelohania contejeani Henneguy \& Thélohan

Sporozoaire indéterminé id.

\section{Sarcosporidia}

Sarcosporidie indéterminée

\section{Haplosporidia}

Urosporidium (gen. ?)

Charletyi R.-Ph. Dollfus 1944

\section{Coccidia}

\section{Eimeriidae}

Eimeria stiedai (Lindemann 1863)

\section{Eimeria sp.}

Cyclospora viperae Marie Phisalix

Caryospora legeri Hoare 1932 Coccidies indéterminées

$$
\text { id. }
$$

localisation Anguilla anguilla (L.) incertaine branchies Gardonus rutilus (L.) vésicule Gardonus rutilus (L.) biliaire branchies Cobitis barbatula $\mathrm{L}$. id. Cobitis barbatula L.

musculature Astacus pallipes Lereboullet 1858

branchies Gardonus rutilus (L.)

id. Perca fluviatilis (L.) (Goeze)

\section{parenchyme Catenotaenia dendritica}

musculature Sorex minutus L. 1766 
id.

id.

Coccidies indéterminées

id.

id.

id.

id.

\section{Hœmosporidia}

Haemoproteus sp.

id.

id.

id.

id.

id.

id.

$i d$.

id.

id.

Leucocytozoon sp.

id.

id.

id.

id.

id.

Plasmodium sp.

id.

Plasmodium sp.

Plasmodium sp.

Plasmodium elongatum Huff 1930

Plasmodium sp.

Plasmodium sp.

Plasmodium sp.

Hématozoaires indéterminés

id.

id.

id. id. Oryctolagus cuniculus (L.), domest.

intestin Oryctolagus cuniculus (L.) feet foie rus

intestin Sciurus vulgaris $\mathrm{L}$.

id. Mustela putorius $\mathrm{L}$.

id. Columba livia Gmel., domest.

id. Gallus gallus (L.), domest.

id. Phasianus colchicus L.

sang Garrulus glandarius L.

id. Turdus philomelos Brehm

id. Turdus merula L.

id. Gecinus viridis L.

id. Athene noctua (Scopoli)

id. Strix aluco L.

id. Accipiter gentilis (L.)

id. Buteo buteo (L.)

id. Falco tinnunculus L.

id. Columba palumbus L.

sang Corvus corone L.

id. Garrulus glandarius L.

id. Pica pica (L.)

id. Athene noctua (Scopoli)

id. Strix aluco L.

id. Meleagris gallopavo L.

sang Corvus corone L.

id. Fringilla coelebs $\mathrm{L}$.

id. Garrulus glandarius L.

id. Passer domesticus (L.)

id. Pica pica (L.)

sang Turdus merula $\mathrm{L}$.

id. Turdus sp.

id. $\quad$ Caccabis rufa (L.)

reins Parus ater $\mathrm{L}$.

sang Athene noctua (Scopoli)

id. Caccabis rufa (L.)

id. Perdix perdix (L.) 


\section{Piroplasma}

Piroplasma achromatica

(Yakimoff 1912)

\section{Toxoplasma}

Toxoplasma sp.

\section{Incerta sedis}

Grahamella talpae 1911

Pirhaemocyton lacertae

E. Brumpt \& G. Lavier 1935

\section{Hœmogregarina}

Hémogrégarines indéterminées

id.

id.

id.

Karyolysus lacertae (Danilewsky 1886)

\section{Gregarina}

\section{Gregarinidae}

Gregarina typographi A.-Gilbert Fuchs 1915

Gregarina munieri (Aimé

Schneider 1875)

$$
\text { id. }
$$

Gregarina acuta (L. Léger 1892)

Gregarina sp.

id.

Cnemidospora lutea Aimé Schneider 1882

\section{Actinocephalidae}

Legeria agilis (Aimé Schneider 1876) sang Chiroptère indéterminé

sang Buteo buteo (L.) id. Lacerta viridis Laurenti, expérimentalement

sang Fringilla coelebs L.

sang Sturnus vulgaris L. 1758

du cœur

sang Lacerta muralis Laurenti

Lacerta viridis Laurenti

id. Lacerta muralis Laurenti

Ips sexdentatus Boerner 1776

Chrysolina staphylea L.

Chrysolina menthastri Suff.

Timarcha goettingensis L.

Trox hispidus (Pontoppidan)

Elaterides non identifiés

Pryonichus ater J.-C. Fabricius

Glomeris marginata Villers 1789

Dytiscus semisulcatus Müller, larve 
Actinocephalus conicus (L. Dufour 1826)

Actinocephalus sp.

\section{Stylocephalidae}

Stylocephalus oblongatus

(Hammerschmidt 1838)

\section{Stenophoridae}

Stenophora iulipusilli (J. Leidy 1853)

\section{Dactylophoridae}

Trichorhynchus pulcher Aimé Schneider 1882

\section{Acanthosporida}

Ancyrophora cervicornis

J. Théodoridès 1955

\section{Ciliophora}

Opalines

$$
\text { id. }
$$

\section{Balantidium sp.}

Trichodinopsis paradoxa Claparède \& Lachmann 1858

Infusoires indéterminés

$$
\text { id. }
$$

Holotriche: Tetrahymena sp.

Clausilocola apostropha Jiři Lom 1959 (1)

Proclausilocola complanata Jiři Lom 1959 (1)

\section{Spirochceta}

\section{Dorcus parallelipipedus (L.)}

Ludius ferrugineus (L.), larve Lucanus cervus $\mathbf{L}$.

Elaterides non identifiés

Opatrum sabulosum (L.)

Cylindroiulus londinensis (Leach)

Scutigera coleoptrata (L.)

Silpha carinata Herbst 1783

intestin Rana dalmatina Ch. Bonap.

id. Rana esculenta L.

intestin Triturus vulgaris (L.)

intestin Cyclostoma elegans (O. F. M.)

terminal

intestin Triturus vulgaris (L.)

rectum Rana esculenta $\mathrm{L}$.

cavité Pisidium amnicum (O. F. Mülpalléale ler 1774)

rein Zonitoïdes sp.

cavité Clausiliidae, gen. sp. ? générale (1)

Spirochètes indéterminés

sang Plecotus auritus (L.)

(1) Bozena Grabda leg. et determin. 


\section{VERS}

\section{TREMATODES}

MONOGENEA

Dactylogyridœ B. Bychovsky 1933

Gen. Dactylogyrus Diesing 1850

D. sp.

branchies Gardonus rutilus (L. 1758)

$\begin{array}{lll}\text { id. } & \text { id. } & \text { Esox lucius L. 1758 } \\ \text { id. } & \text { id. } & \text { Tinca tinca } \text { (L. 1758) }\end{array}$

Tetraonchidae B. Bychowsky 1937

Gen. Tetraonchus Diesing 1850

T. monenteron (G. R. Wagener branchies Esox lucius L. 1758 1857)

Gyrodactylidae (P. J. Van Beneden et E. Hesse 1863)

Gen. Gyrodactylus A. v. Nordmann 1832

Gyrodactylus sp. branchies Cobitis barbatula L. 1758

Discocotylidae E. Price 1936

Gen. Diplozoon A. v. Nordmann 1832

D. paradoxum A. v. Nordmann branchies Phoxinus phoxinus (L. 1758) 1832

id. $\quad$ id. Scardinius erythrophthalmus

(L. 1758)

Abramis brama (L. 1758)

Alburnus alburnus (L. 1758)

Blicca bjoerkna (L. 1758)

Polystomatidae Gamble 1896

Gen. Polystoma Zeder 1800

P. integerrimum (Frölich 1791) vessie Rana dalmatina $\mathrm{Ch}$. Bonap.

DIGENEA

GASTROSTOMATA

Bucephalidae F. Poche 1907

Gen. Bucephalus K. v. Baer 1827

B. polymorphus K. v. Baer intestin Esox lucius L. 1758 1827

PROSOSTOMATA

Distome énigmatique R.-Ph. vésicule Tinca tinca (L. 1758) Dollfus 1948 biliaire 
Allocreadiidae M. Stossich 1904

Gen. Allocreadium A. Looss 1900

A. isoporum (A. Looss 1894) tube Leuciscus cephalus (L. 1758) digestif

Gen. Crepidostomum M. Braun 1900

Cr. sp.

$\begin{array}{cl}\text { tube } & \text { Salmo irideus W. Gibbons } 1855 \\ \text { digestif } & \text { (2) }\end{array}$

Sphœrostomatidœe G. S. Thapar \& J. Dayal 1934

Gen. Sphaerostoma Rudolphi 1809

S. bramae (O. F. Müller 1776) intestin Leuciscus cephalus (L. 1758)

Coitoca cidae Y. Ozaki 1928

Gen. Nicolla L. W. Wisniewski 1933

Nicolla gallica (R.-Ph. Dollfus intestin Cottus gobio L. 1758 (nat. et 1941) (3) expériment.)

Anguilla anguilla (L. 1758) (expériment.)

Salmo irideus W. Gibbons 1855 (expériment.)

Deropristida K. I. Skrjabin 1958

Gen. Deropristis T. Odhner 1902

D. inflata (R. Molin 1859) intestin Anguilla anguilla (L. 1758) (4)

Halipegida F. Poche 1926

Gen. Halipegus A. Looss 1899

H. ovocaudatus (Vulpian 1859) sous Rana esculenta L. 1758

la langue

Monorchiidae T. Odhner 1911

Gen. Asymphylodora A. Looss 1899

A. tincae (Modeer 1790) intestin et Tinca tinca (L. 1758) vésicule

biliaire

Gorgoderida A. Looss 1901

Gen. Gorgodera A. Looss 1899

Gorgodera sp.

vessie Rana esculenta L. 1758

(2) Chez une truite arc-en-ciel d'élevage, achetée à Tours.

(3) Correspond presque certainement à Cercaria myzura A. Pagenstecher 1881.

(4) Chez une anguille des environs de Châtellerault. 
Gen. Phyllodistomum M. Braun 1899

Phyllodistomum sp.

intestin Tinca tinca (L. 1758)

Lepodermatidae T. Odhner $1910=$ Plagiorchiida (M. Lühe 1901)

S. f. LEPODERMATINAE A. Looss $1899=$

PLAGIORCHIINE (M. Lühe 1901)

Gen. Lepoderma A. Looss 1899 = Plagiorchis M. Lühe 1899

$\boldsymbol{P}$. vespertilionis (O. F. Müller 1784)

P. cirratus (Rud. 1802)

P. elegans (Rud. 1802) (4 $4^{\text {bion }}$

Plagiorchis sp.

Plagiorchis sp.

Plagiorchis sp. (5)

P. maculosus (Rudolphi 1802)

Plagiorchis sp.

P. hirundinis (Froelich 1791)

P. lacertae (Rudolphi 1819)

id.

id.

Plagiorchis cf. mentulata (Rudolphi 1819)

Plagiorchis sp.
Eptesicus serotinus (Schreber 1775)

Myotis emarginatus (Et. Geoffroy-St-Hilaire 1806)

Myotis myotis (Borkhausen 1797)

Pipistrellus pipistrellus (Schreber 1775)

Plecotus auritus (L. 1758)

Rhinolophus ferrumequinum

(Schreber 1774)

Rhinolophus hipposideros

(Bechstein 1800)

intestin Mus musculus L. 1758 albus. (expérimen-

talement)

id.

Mus musculus L. 1758 et $M$. musculus albus

intestin Mus alexandrinus Is. GeoffroySt-Hilaire 1812

intestin Rattus rattus (L. 1758)

intestin Merula merula (L. 1758)

intestin Chelidon rustica (L. 1758)

Chelidon rustica (L. 1758)

Hirundo (Delichon) urbica $\mathbf{L}$. 1758

intestin Lacerta agilis L. 1758

id. Lacerta sp.

id. Lacerta muralis Laurenti 1768

intestin Lacerta viridis Laurenti 1768

id. Athene noctua (Scopoli 1769)

(5) Dimensions des œufs sur le vivant : $34,48 \times 22,412 ; 36,204 \times 24,136$; $37,928 \times 22,412 ; 39,652 \times 18,964 ; 41,376 \times 22,412 ; 43,10 \times 24,136 u$.

$\left(4^{\text {bis }}\right)$ fide E. Styczynska-Jurewicz (1961). 
Gen. Paralepoderma R.-Ph. Dollfus 1950

P. cloacicola (M. Lühe 1899) cloaque Natrix natrix (L. 1758) (6)

P. brumpti (A. Buttner 1950) musculature Triturus helveticus (Razou$\left(6^{\text {bis }}\right)$ mowsky 1789) (métacercaire enkystée dans la nature et expérimentalement)

musculature Alytes obstetricans (Laurenti 1768) (métacercaires enkystées dans la nature et expérimentalement)

S. f. PNEUMONOECEINAE (Mehra 1937)

= Haematoloechinae Freitas \& Lent 1939

Gen. Pneumonoeces A. Looss 1902 = Haematoloechus A. Looss 1899

P. variegatus (Rud. 1819) poumons Rana esculenta L. 1758

S. f. OPISTHIOGLYPHINAE R.-Ph. Dollfus 1949

Gen. Opisthioglyphe A. Looss 1899

O. ranae (Froelich 1791)

intestin Rana esculenta L. 1758

intestin Triturus helveticus (Razoumowsky 1789), adulte dans têtard

intestin Alytes obstetricans Laurenti grêle 1768 (expérimentalem. chez et rectum têtards)

musculature Alytes obstetricans Laurenti 1768 (métacercaires enkystées, expérimentalement et dans la nature)

intestin Bufo bufo (L. 1758)

Opisthioglyphe sp. intestin Rana dalmatina Ch. Bonap. 1839

O. endoloba (F. Dujardin 1845) intestin Rana esculenta L. 1758

S. g. Lecithopyge Perkins 1928

O. (L.) rastellus (P. Olsson poumons Rana esculenta $\mathrm{L} .1758$ 1876)

(6) Dimensions des œufs sur le vivant : 29-30 $\times 16-17 u$.

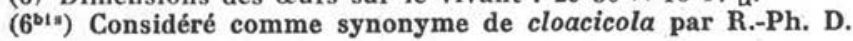




\section{S. f. HAPLOMETRINAE Pratt 1902}

Gen. Haplometra A. Looss 1898

H. cylindracea (Zeder 1800) poumons Rana esculenta L. 1758

Gen. Saphedera A. Looss 1902 = Macrodera A. Looss 1899

S. longicollis (Abildgaard 1788)

Natrix natrix (L. 1758)

$=S$. naja (Rudolphi 1819)

Natrix maura (L. 1758)

\section{S. f. LEPTOPHALLINAE J. Dayal 1938}

Gen. Leptophallus M. Lühe 1909

L. nigrovenosus (Bellingham bouche et Natrix natrix (L. 1758) 1844)

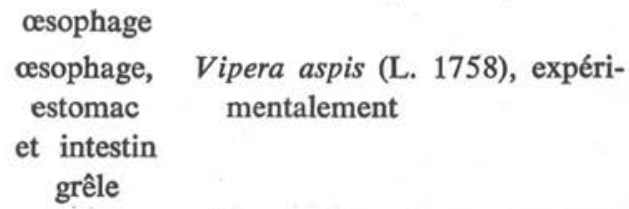

Alytes obstetricans Laurenti 1768 (métacercaire, enkystement expérimental et dans la nature chez des têtards)

Telorchiidae W. Nicoll 1924

S. f. TELORCHIINAE A. Looss 1899

Gen. Telorchis Lühe 1899-Looss 1899

T. assula (F. Dujardin 1845) $=\quad$ intestin Natrix natrix (L. 1758)

T. ercolanii (F. Monticelli

1893)

Natrix maura (L. 1758)

Cephalogonimidae W. Nicoll 1913

S.f. Cephalogoniminæ A. Looss 1899

Gen. Cephalogonimus J. Poirier 1886

C. europaeus L. Blaizot 1910 intestin Rana esculenta L. 1758

(7) grêle

(7) Dimensions des œufs $(u): 28,1 \times 19,5 ; 33,49 \times 16,7 ; 33,49 \times 18$. D'après ces dimensions, il ne peut pas s'agir de Cephalogonimus retusus (F. Dujardin 1845 ), dont les œufs mesurent $54 \times 36$, d'après Dujardin. 
Prosthogonimidae F. Lahille 1922

S.f. Prosthogoniminæ Max Lühe 1899

Gen. Prosthogonimus M. Lühe 1899

P. ovatus (Rudolphi 1803)

bourse de Corvus corone L. 1758

Fabricius

Merula merula (L. 1758)

Athene noctua (Scopoli 1769)

Garrulus glandarius (L. 1758)

Gallinula chloropus (L. 1758)

Prosthogonimus sp.

bourse de Corvus corone L. 1758

Fabricius

Phylloscopus rufus Bechstein 1795

Sturnus vulgaris L. 1758

Anas platyrhynchos L. 1758, fer.

Tringa ochropus L. 1758

Rallus aquaticus L. 1758

P. brauni K. I. Skrjabin 1919

id. Pica pica (L. 1758)

Garrulus glandarius (L. 1758)

S. f. Omphalometrinae A. Looss 1899

Gen. Omphalometra A. Looss 1899

O. flexuosa (Rudolphi 1809) intestin Talpa europaea L. 1758

Lecithodendriidae T. Odhner 1910

S. f. Lecithodendriinæ Max Lühe 1901

Gen. Lecithodendrium A. Looss 1896

Lecithodendrium sp. intestin

Rhinolophus hipposideros

(Bechstein 1800)

Lecithodendrium linstowi intestin Pipistrellus kuhli (Natterer in

R.-Ph. Dollfus 1931

Kuhl 1817)

Gen. Prosthodendrium R.-Ph. Dollfus 1931

P. chilostomum (Mehlis 1831)

Rhinolophus hipposideros

(Bechstein 1800)

Prosthodendrium sp.

Plecotus auritus (L. 1758)

Gen. Laterotrema V. D. Semenov 1927

L. arenula (F.-C.-H. Creplin intestin Gallinula chloropus (L. 1758) 1825)
S. f. Pycnoporinae
S. Yamaguti 1958

Gen. Pycnoporus A. Looss 1899

P. heteroporus (F. Dujardin intestin 1845)

Pipistrellus kuhli (Natterer in Kuhl 1817) 
S. f. Leyogoniminæ R.-Ph. Dollfus 1951

Gen. Leyogonimus T. G. Gynezinskaïa in Skrjabin 1948

L. polyoon (O.v. Linstow intestin Gallinula chloropus (L. 1758) 1887)

S. f. Eumegacetinoe Mehra 1935

Gen. Eumegacetes A. Looss 1900

E. emendatus Max. Braun 1901 intestin Caprimulgus europaeus L. 1758

S. S. Pleurogeninae (A. Looss 1899) L. Travassos 1921

Gen. Pleurogenes A. Looss 1899

P. claviger (Rudolphi 1819) intestin Rana esculenta L. 1758

Gen. Pleurogenoïdes L. Travassos 1921

P. medians (P. Olsson 1876) intestin Rana esculenta L. 1758

Gen. Prosotocus A. Looss 1899

P. fülleborni L. Travassos 1930 intestin Rana esculenta L. 1758

P. confusus (A. Looss 1894) Rana esculenta L. 1758

Prosotocus sp. intestin Rana esculenta L. 1758

Gen. Brandesia M. Stossich 1899

B. turgida (Brandes 1888) pylore Rana esculenta L. 1758

Heterophyidae T. Odhner 1914

Gen. Euryhelmis Poche 1926

E. squamula (Rud. 1819) intestin grêle Mustela putorius L. 1758

Dicrocœliidae T. Odhner 1910
S. f. Dicrocoeliinae
A. Looss 1899

Gen. Dicrocoelium F. Dujardin 1845

D. soricis (Diesing 1858 ex. vésicule Sorex araneus L. 1758 Pontaillié 1853) biliaire

Gen. Platynosomum A. Looss 1907

P. alectoris W. Nöller \&

Caccabis rufa (L. 1758)

K. Enigk 1932

$\begin{array}{lll}\text { P. acuminatum W. Nicoll } & \text { vésicule } & \text { Strix aluco L. } 1758 \\ 1915 & \text { biliaire } & \end{array}$

Gen. Conspicuum G. D. Bhalerao 1936

C. sp.

vésicule Turdus merula L. 1758

biliaire

Gen. Lyperosomum A. Looss 1899

L. vitta (F. Dujardin 1845) en partie Apodemus sylvaticus (L. 1758), dans le canal pancréatique

en partie

dans l'intestin 
intestin Mus alexandrinus Is. GeoffroySt-Hilaire 1812

Lyperosomum longicauda (Ru- vésicule Corvus corone L. 1758 dolphi 1819)

Lyperosomum sp.

Lyperosomum sp.

Lyperosomum sp.

Lyperosomum sp.

biliaire

vésicule Pica pica (L. 1758)

biliaire

pancréas Pica pica (L. 1758)

vésicule Turdus ericetorum Turton

biliaire $\quad 1807$

et canal

biliaire

voies Accipiter gentilis (L. 1758)

biliaires

Gen. Oswaldoia L. Travassos 1919

S. g. Dicrocoelioides R.-Ph. Dollfus 1954 (gen.)

O. (D.) petiolata (A. Railliet vésicule Garrulus glandarius (L. 1758) 1900)

biliaire Otis (Tetrax) tetrax (L. 1758)

O. (D.) skrjabini (Soloviov 1911)

canaux Corvus corone L. 1758

biliaires Pica pica (L. 1758)

O. (D.) sp.

id.

Gallinula chloropus (L. 1758)

Gen. Brachylecithum J. K. Strom 1940

B. alfortense (A. Railliet mss) foie Pica pica (L. 1758) in R.-Ph. Dollfus 1954

B. attenuatum (F. Dujardin foie Turdus merula L. 1758 1845)

Brachylecithum sp.

canaux Oriolus oriolus (L. 1758)

du foie

Brachylecithum sp. (8) (fig. 14) id. Garrulus glandarius (L. 1758)

Opisthorchiidce (M. Lühe 1901) M. Braun 1901

S. f. Metorchilinae M. Lühe 1908

Gen. Metorchis A. Looss 1899

M. xanthosomus (F.-C.-H.

Creplin 1846) vésicule Gallus gallus (L. 1758), domest. biliaire

intestin et Anas platyrhynchos L. 1758, vésicule domest.

biliaire

vésicule Anas platyrhynchos L. 1758, biliaire domest. expérimentalement

(8) Longueur, $3,6 \mathrm{~mm}$. ; largeur au niveau du testicule postérieur, $0,31 \mathrm{~mm}$., ventouse orale, $185-190 u$; ventouse ventrale, $190-195 \mu$; pharynx, $51 u$; testicule antérieur, $215 \times 251 \mathrm{u}$; testicule postérieur, $240 \times 256 \mu$; ovaire, $128 \times 185 \mu$; œufs, $41,7 \times 22,1$ à $25 u$. 
id. Anser anser (L. 1758), domest. expérimentalement

musculature Cobitis (Nemachilus) barbatula L. 1758, métacercaires enkystées

M. albidus (M. Braun 1893) vésicule Neomys fodiens (Pallas 1756) biliaire

Felis catus L. 1758 (expérimentalement)

musculature Gobio gobio L. 1758, métacercaires enkystées

Gen. Pseudamphistomum M. Lühe 1908

P. truncatum (Rud. 1819) var. vésicule Lutra lutra (L. 1758) lutrae $\mathrm{R} .-\mathrm{Ph}$. Dollfus $1936 \quad$ biliaire

Pseudamphistomum sp. vésicule Mustela putorius L. 1758 biliaire et cholédoque

Microphallidae L. Travassos 1921

Gen. Levinseniella Ch. W. Stiles \& A. Hassall in H. B. Ward 1901
L. (gen. ?) sp.
intestin
Tringa ochropus L. 1758

Orchipedidae K. I. Skrjabin 1924

Gen. Orchipedum M. Braun 1901

O. isostoma (Rudolphi 1819)

fosses Canis familiaris L. 1758 (expénasales rimentalement)

Felis catus L. 1758, domest. (expérimentalement)

Mustela nivalis L. 1766 (expérimentalement)

Mustela putorius L. 1758 (expérimentalement)

Mustela putorius furo L. 1758 (expérimentalement)

Vulpes vulpes (L. 1758) (expérimentalement)

cavité Astacus pallipes Lereboullet

générale 1858, métacercaire 
Eucotylidae K. I. Skrjabin 1924

Gen. Tamerlania K. I. Skrjabin 1924

T. zarudnyi K. I. Skrjabin 1924 reins Turdus merula (L. 1758)

$=T$. gallica R.-Ph. Dollfus 1946

\begin{tabular}{|c|c|}
\hline reins & Corvus corone L. 1758 \\
\hline $\begin{array}{l}\text { reins } \\
\text { et foie }\end{array}$ & $\begin{array}{l}\text { Garrulus glandarius (L. 1758) } \\
(9)\end{array}$ \\
\hline reins & Pica pica (L. 1758) \\
\hline reins & $\begin{array}{l}\text { Turdus ericetorum Turton } \\
1807\end{array}$ \\
\hline & $\begin{array}{cl}\text { Coloeus monedula } \\
(\text { Brehm } 1831)\end{array}$ \\
\hline
\end{tabular}

\section{Psilostomatidae (Thapar et Lal 1935)}
S. f. Psilostomatinæ
(A. Looss 1900)

Gen. Psilostomum A. Looss 1899

Psilostomum sp.

intestin Gallinula chloropus (L. 1758),

Gen. Psilotrema T. Odhner 1913

P. oligoon (O. v. Linstow 1887) intestin Gallinula chloropus (L. 1758)

Leucochloridiidae R.-Ph. Dollfus 1934

S. f. Leucochloridiince F. Poche 1907

Gen. Leucochloridium Carus 1835

L. macrostomum (Rud. 1803) intestin Gallinula chloropus (L. 1758) postérieur

Saxicola rubetra (L. 1758)

Leucochloridium sp. bourse de Rallus aquaticus L. 1758 Fabricius

Leucochloridium sp. cæa $\quad$ Rallus aquaticus L. 1758

Brachylœmidœ Ch. Joyeux \& Foley 1930

Gen. Brachylaema F. Dujardin $1843\left(9^{\text {bis }}\right)$

B. cf. fulva F. Dujardin 1843 estomac Sorex araneus L. 1758

(9) Dans les reins d'un même Geai, jusqu'à 56 exemplaires.

(9 $\left.9^{\text {is }}\right)$ Pour les hôtes des larves, voir p. 245, 246-249. 
Brachylaema sp.

B. erinace $\mathrm{Em}$. Blanchard 1847

$B$. erinacei spinosulus (K. Hofmann 1899)

B. advena F. Dujardin 1843 intestin (10)

B. recurva F. Dujardin 1845

B. arcuata F. Dujardin 1845

B. arcuata F. Dujardin 1845 (11)

Brachylaema sp.

Brachylaema sp.

Brachylaema sp.

Brachylaema sp. intestin Crocidura russula (Hermann 1780)

intestin Erinaceus europaeus L. 1758

intestin Erinaceus europaeus L. 1758

intestin Turdus philomelos Brehm 1831

intestin Eliomys quercinus (L. 1766)

intestin Turdus philomelos Brehm 1831

id. $\quad$ Turdus merula L. 1758

Garrulus glandarius (L. 1758)

intestin Tyto alba (Scopoli 1769) (expérimentalement)

intestin Strix aluco L. 1758 (expérimentalement)

intestin Strix aluco L. 1758

intestin Columba palumbus L. 1758

grêle

Gen. Ityogonimus M. Lühe 1899

I. lorum (F. Dujardin 1845)

intestin Talpa europaea L. 1758

(10) Dimensions de 2 immatures.

Epines cuticulaires absentes, peut-être tombées

\begin{tabular}{|c|c|c|c|}
\hline Longueur & & $1,211 \mathrm{~mm}$. & $1,257 \mathrm{~mm}$. \\
\hline Largeur . & 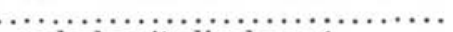 & $0,434 \mathrm{~mm}$. & $0,434 \mathrm{~mm}$. \\
\hline Ventouse & $\begin{array}{c}\text { orale longitudinalement } \ldots \ldots \ldots \\
\text { transversalement } \ldots \ldots \ldots\end{array}$ & $\begin{array}{l}172,41 \mu \\
189,65 \mu\end{array}$ & $\begin{array}{l}189,65 \mu \\
189,65 \mu\end{array}$ \\
\hline Ventouse & ventrale $\ldots \ldots \ldots \ldots \ldots \ldots \ldots \ldots$ & $162 \mu$ & $182,75 \mu$ \\
\hline Pharynx & $\begin{array}{l}\text { longitudinalement } \\
\text { transversalement }\end{array} \ldots \ldots \ldots \ldots \ldots \ldots \ldots$ & $\begin{array}{l}103,4 \\
103,4\end{array}$ & $\begin{array}{l}103,4 \\
113,8 \\
\mu\end{array}$ \\
\hline Esophage & 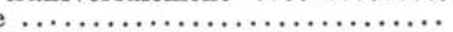 & $44,82_{\mu}$ & $24 u$ \\
\hline
\end{tabular}

(11) Cuticule finement spinulée antérieurement : longueur, 1,6 mm. ; largeur, $0,5 \mathrm{~mm}$; ; ventouse orale, $208 \times 201,5 \mu$; ventouse ventrale, $221 \times 234 \mathrm{u}$; pharynx, $117 \times 149,5$ u ; œufs, $27,58 \times 17,24$ แ. Vitellogènes s'étendant du pore génital presque jusqu'au niveau du bord antérieur de l'acétabulum. Utérus dépassant antérieurement l'acétabulum. Testicule antérieur orbiculaire. Testicule postérieur de forme irrégulière, à peine un peu lobé. Ovaire de forme irrégulière. Pore génital à droite de l'ovaire, légèrement postérieur au bord antérieur du testicule antérieur.

Un autre individu de la même récolte a la ventouse orale plus grande que la ventrale : ventouse orale, $216 \times 180 u$, ventrale, $196 \times 168 u$; pharynx, $120 \times 116 u$. Du côté droit, les vitellogènes n'atteignent pas le bord postérieur de l'acétabulum ; du côté gauche, ils l'atteignent à peine. L'utérus atteint presque le pharynx. Les glandes génitales sont presque régulièrement circulaires. (Eufs, $27,58 \times 17,24-18 u$.

Les dimensions des œufs sont très variables ; chez un troisième individu, on trouve $31,03 \times 19,82 u$ à $32,75 \times 20,68 u$; chez un quatrième : $34,48 \times 18,96 u$ à $36,20 \times 15,51-17,24$ u. R.-Ph. D. 
Echinostomatidae (A. Looss 1902)
S. f. Echinostomatinae
(A. Looss 1899) $\left(11^{\text {bis }}\right)$

Gen. Echinostoma Rudolphi 1809

E. revolutum (Froelich 1802)

bourse de Anas platyrhynchos L. 1758 Fabricius domest.

intestin Anas platyrhynchos L. 1758 (expérimentalement)

intestin Gallus gallus (L. 1758) domest. (expérimentalement)

reins Rana esculenta L. 1758 (métacercaires enkystées chez têtards, expérimentalement)

E. cf. pungens Linstow 1894 intestin Gallinula chloropus (L. 1758) (12)
branchies et Gasterosteus aculeatus L. 1758: œsophage (métacercaires enkystées)

Gen. Echinoparyphium Dietz 1909

E. recurvatum (O. v. Linstow intestin Anas platyrhynchos L. 1758 . 1873)

domest.
Anas platyrhynchos L. 1758
domest. (expérimentalement).
Anser anser (L. 1758) domest.
(expérimentalement)
Gallus gallus (L. 1758) domest..
(expérimentalement)
musculature Rana esculenta L. 1758, méta--
cercaires enkystées expéri-.
mentalement

Gen. Echinochasmus Dietz 1909

E. beleocephalus (O. v. Lins- intestin Ardea cinerea L. 1758 tow 1873) (13) grêle

(12) 32 crochets céphaliques. Dorsalement, 23 grands crochets sur deux rangs. A droite 5 grands crochets angulaires; à gauche 2 grands crochets angulaires + 2 petits. CEufs, 97,29 à $101,52 u \times 57,105 u$. Maximum, $105,75 \times 59,22 u$. 7 u.

(13) Petits spécimens portant 24 crochets céphaliques longs de $28_{u}$, larges de-

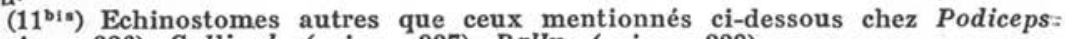
(voir p. 226), Gallinula (voir p. 227), Rallus (voir p. 228). 
Gen. Hypoderaeum Dietz 1909

H. conoideum (M.-El. Bloch intestin 1782)

Gen. Episthmium M. Lühe 1909

Episthmium sp. (14)

bourse de Ardea cinerea L. 1758

Fabricius,

gros intestin

Cyclocœlidœe W. Kossack 1910

S. f. Cyclocoelinae M. Stossich 1902

Gen. Cyclocoelum Brandes 1892

S. g. Cyclocoelum R.-Ph. D. 1948

C. (C.) mutabile (Zeder 1800) sacs aériens Gallinula chloropus (L. 1758)

S. f. Hyptiasminae R.-Ph. Dollfus 1948

Gen. Hyptiasmus W. Kossack 1911 S. g. Hyptiasmus R.-Ph. D. 1948

$H$. $(H$.$) brumpti \mathrm{R} .-\mathrm{Ph}$. Dollfus cavité Gallinula chloropus (L. 1758) 1948

thoracique

sinus

orbitaires

$\begin{array}{lll}\text { S. f. Typhlocoelinae } & \text { E. C. Harrah } 1922\end{array}$

Gen. Typhlocoelum M. Stossich 1902

T. cymbium (Diesing 1850) trachée Anas platyrhynchos L. 1758 fer.

Notocotylidae M. Lühe 1909

Gen. Notocotylus Diesing 1839

N. attenuatus (Rudolphi 1809) bourse de Anas platyrhynchos L. 1758 Fabricius domest.

et intestin

Notocotylus sp. $\quad$ intestin Gallinula chloropus (L. 1758)

Gen. Catatropis T. Odhner 1905

Catatropis sp.

C. verrucosa (Froelich 1789) cæca Gallinula chloropus (L. 1758)

cæca Gallus gallus (L. 1758) domest.

(14) Corps spinulé jusqu'à l'extrémité postérieure. 22-24 crochets céphaliques longs de $42 \mu$. Vitellogènes atteignant antérieurement la ventouse orale. 
Paramphistomatidae Fischoeder 1901
S. f Diplodiscinae
L. Cohn 1904

Gen. Diplodiscus Diesing 1836

$\begin{array}{cc}\text { D. subclavatus (Pallas 1760) } & \begin{array}{c}\text { rectum } \\ \text { et cloaque } \\ \text { vessie }\end{array}\end{array}$

Gen. Opisthodiscus L. Cohn 1904

O. diplodiscoïdes L. Cohn 1904 cloaque Rana esculenta L. 1758

Schistosomatidae A. Looss 1899

Gen. Bilharziella A. Looss 1899

Furcocercaire à 2 ocelles (dermatite humaine)

Bilharziella sp. masse Limnaea auricularia (L. 1758) viscérale

vaisseaux Anas platyrhynchos L. 1758 mésenté- (expérimentalement)

\section{STRIGEIDA}

Strigeidce A. Railliet 1919

Gen. Strigea Abildgaard 1793
S. strigis (Schrank 1788)
intestin Strix aluco L. 1758
S. falconis L. Szidat 1928
intestin Buteo buteo (L. 1758)
Pernis apivorus (L. 1758)
S. falconis-palumbi Viborg intestin Buteo buteo (L. 1758) 1795
Gen. Uvulifer S. Yamaguti 1934
U. denticulatus (Rudolphi tube Alcedo atthis (L. 1758) 1819) digestif

Gen. Cotylurus L. Szidat 1928

C. cornutus (Rudolphi 1809) gros intestin Anas platyrhynchos L. 1758 domest.
C. hebraicus G. Dubois 1934 tube Gallinula chloropus (L. 1758) digestif

Gen. Apatemon L. Szidat 1928

A. gracilis (Rudolphi 1819)

Anas platyrhynchos L. 1758 domest. 


\section{Diplostomatidae (J. Poirier 1886)}

Gen. Neodiplostomum A. Railliet 1919

N. krausei G. Dubois 1937 var. intestin Buteo buteo (L. 1758) ovata G. Dubois 1938

N. (N.) attenuatum (O. v. Lins- intestin Buteo buteo (L. 1758) tow 1906) *

Gen. Posthodiplostomum G. Dubois 1936

P. cuticola (Al. v. Nordmann intestin Ardea cinerea L. 1758 1832)

branchies Gardonus rutilus (L. 1758)

Gen. Tylodelphis M. Diesing 1850

T. clavata (Al. v. Nordmann intestin Ardea cinerea L. 1758 1832)

Gen. ?

Tetracotyle phoxini E.-C. Faust cerveau Phoxinus phoxinus (L. 1758) 1918

intestin Anas platyrhynchos L. 1758 domest. (adulte expérimentalement)

Tetracotyle sp., pl. libres et en- musculature Cobitis (Nemachilus) barbatula kystés

L. 1758

Cyathocotylidae F. Poche 1925

Gen. Cyathocotyle P. Mühling 1896

C. prussica P. Mühling 1896 intestin Gallinula chloropus (L. 1758)

\section{CESTODES}

PSEUDOPHYLLIDEA Carus 1863

Caryophyllaidae Rud. Leuckart

Gen. Caryophyllaeides O. Nybelin 1922

Caryophyllaeides fennica

(Guido Schneider 1902)

(fig. 63)

intestin Abramis brama (L. 1758)

Blicca bjoerkna (L. 1758)

Leuciscus cephalus (L. 1758)

Leuciscus leuciscus (L. 1758)

Leuciscus rutilus (L. 1758)

Scardinius erythrophthalmus

(L. 1758)

* Détermination par le $\mathrm{D}^{\mathrm{r}}$ Georges Dubois.

AnN. de Parasitologie, T. XXXVI, N ${ }^{\circ} 3,1961$. 
Bothriocephalidae Th. Sp. Cobbold 1864 (Ptychobothriidae Max Lühe 1902)

Bothriocephalus claviceps intestin Anguilla anguilla (L. 1758)

(Goeze 1782)

Diphyllobothriidœ Max Lühe 1910

Schistocephalus gasterostei cavité Gasterosteus aculeatus L. 1758

(O. Fabricius 1780)

$=S$. solidus $(\mathrm{O}$. F. Müller générale 1776)

Diphyllobothrium sp. œufs dans Lutra lutra (L. 1758) les selles

Diphyllobothrium sp. cavité Macrocyclops albidus (Jurine générale 1820), expérimentalement

CYCLOPHYLLIDEA P. J. Van Beneden

\section{Amabiliidae B. H. Ransom 1909}

Tatria cf. acanthorhyncha (K. intestin Podiceps ruficollis Pallas 1764 Wedl 1855) (15) (fig. 64)

Fig. 64. - Crochet (long de 17-22 $\omega$ ) de Tatria sp. Les ventouses ont un diamètre moyen de $0,114-0,116 \mathrm{~mm}$. Richelieu, 16-9-1955. Intestin de Podiceps ruficollis (Pallas 1764).

Il y a 14 crochets, comme chez $T$. acanthorhyncha (Wedl 1855), mais, chez acanthorhyncha, ils n'ont pas la même forme.

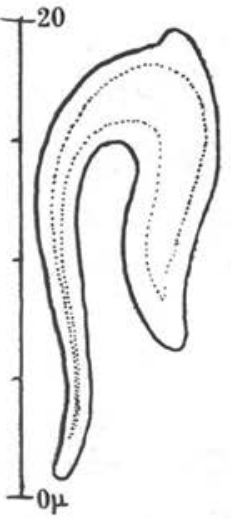

Tatria sp.

intestin Gallinula chloropus (L. 1758)

Schistotaenia macrorhynchus intestin Podiceps ruficollis Pallas 1764 (Rudolphi 1810) (16)

\section{Anoplocephalidœe O. Fuhrmann 1907}

Aporina delafondi (A. Railliet intestin Columba palumbus L. 1758 1892)

(15) 14 crochets de $23 u$.

(16) 20 crochets de $145 u$. 
Cittotaenia leuckarti (G. intestin Oryctolagus cuniculus

Riehm 1881) (17)

Cittotaenia pectinata (Goeze intestin 1782)

Cittotaenia denticulata (Rud. intestin 1804) (18)

Cittotaenia sp. juv. immature

Paranoplocephala blanchardi (R. Moniez 1891) (19)

Paranoplocephala blanchardi

(R. Moniez 1891) (20)
(L. 1758), ferus

Oryctolagus cuniculus

(L. 1758), ferus

Oryctolagus cuniculus

(L. 1758), ferus

intestin Lepus europaeus Pallas 1778

intestin Evotomys (Clethrionomys) gla. reolus (Schreber 1792)

intestin Microtus agrestis (L. 1761)

\section{Nematotœniidas Max Lühe 1910}

Nematotaenia dispar (Goeze intestin Bufo bufo (L. 1758) 1782)

\section{Davaineida M. Braun 1900}

Davainea sp. Larve cysticercoïde enkystée

Raillietina (Skrjabinia) sp.

Raillietina (Skrjabinia) sp.

Raillietina frontina (F. Dujardin 1845) (21)

Idiogenes otidis H. Krabbe intestin Tetrax tetrax (L. 1758)
Agriolimax sp.

intestin Caccabis rufa (L. 1758)

intestin Perdix perdix (L. 1758)

intestin Gecinus viridis (L. 1758)

(17) Plus grande largeur, $7 \mathrm{~mm}$. Ventouses, $152-163 u$. Poche du cirre, $157 \times 70 u$; $169 \times 62_{\mu} ; 154 \times 77 \mu$. Quelques testicules (diamètre $70 \mu$ ) dans la partie médiane du bord postérieur du proglottis, le plus grand nombre en deux groupes latéraux de chacun 32 à 35 . Un immature, long de $16 \mathrm{~mm}$., large de $2 \mathrm{~mm}$., a des ventouses de $305 \mu$.

(18) Chez deux spécimens, diamètre des ventouses, 0,325 à $0,287 \mathrm{~mm}$. Plus grande largeur du strobile, $15 \mathrm{~mm}$. Poche du cirre, $0,875 \times 0,300$ et $0,925 \times 0,270$. Diamètre des testicules très variable : 0,$06 ; 0,05625 ; 0,0918 ; 0,090 \times 0,1125$; $0,10125 \times 0,1125$. Eufs, diamètre environ $60 \mu$. Le cirre est entièrement spinulé. Chez un autre spécimen, la poche du cirre est un peu plus petite : $076 \times 0,25 \mathrm{~mm}$.

(19) Diamètre des ventouses, 0,40-0,55 mm. Poche du cirre, $215 \times 81-97$ à $220 \times 86 \mu$. (Eufs, 34,6 $\mu$.

(20) Longueur, $63 \mathrm{~mm}$.; plus grande largeur, $3,5 \mathrm{~mm}$. Scolex, diamètre, 0,8322. Ventouses, $0,172 \times 0,189$ à $0,189 \times 0,189$. Poche du cirre, $342-345 \times 115-124$ ib 30-40 testicules dépassant antiporalement le vaisseau excréteur ventral.

(21) Nous avons trouvé jusqu'à environ 300 individus dans l'intestin moyen. R.-Ph. 1). 

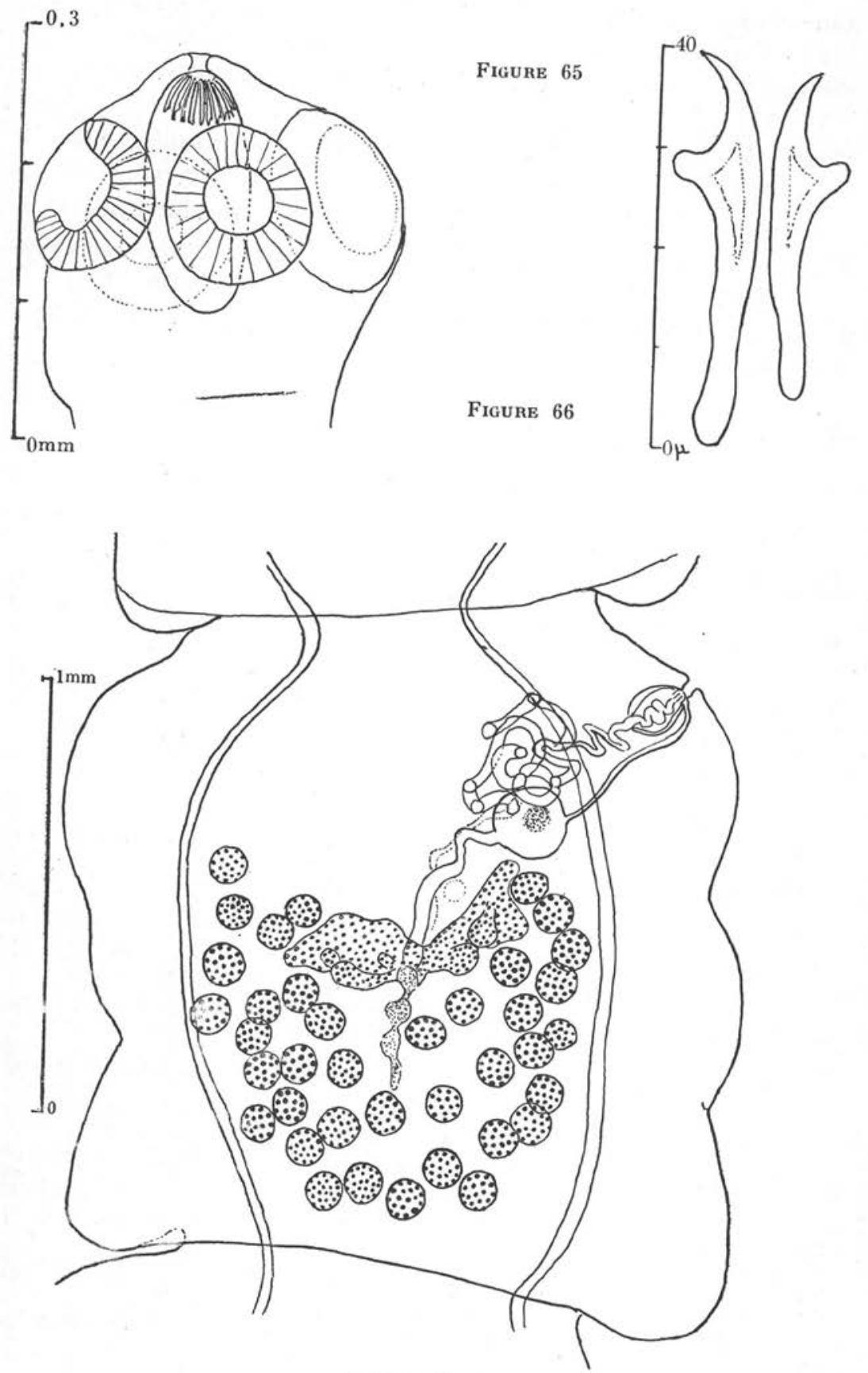

Figure 67 
Dilepidida A. Railliet \& A. Henry 1909

Anomotaenia depressa (aucto- intestin Apus apus (L. 1758) rum) (22)

Anomotaenia sp. cf. globulus intestin Tringa ochropus L. 1758

(C. Wedl 1855)

Anomotaenia $\mathrm{cf}$. arionis (Th. v. Siebold 1850)

Anomotaenia cf. trigonocephala

(H. Krabbe 1869)

Anomotaenia sp.

Anomotaenia sp.

Dilepis brachyarthra Cholodkovsky 1906 (23)

Dilepis undula (Schrank 1788) (24) (fig. 68)

\begin{tabular}{|c|c|}
\hline $\begin{array}{c}\text { cavité } \\
\text { yiscérale }\end{array}$ & Arion rufus L. 1758 \\
\hline intestin & Pica pica (L. 1758) \\
\hline intestin & Sitta europaea L. 1758 \\
\hline intestin & Merula meruīa (L. 1758) \\
\hline intestin & Corvus corone L. 1758 \\
\hline intestin & $\begin{array}{l}\text { Turdus ericetorum Turton } \\
1807\end{array}$ \\
\hline intestin & Turdus viscivorus L. 1758 \\
\hline
\end{tabular}

(22) Crochets longs de $43 \mathrm{u}$. Poche du cirre, $325 \times 98^{\prime} \mathrm{u}$. Les testicules sont au nombre de 16-21; cependant, quand les testicules sont gros (diam. 40-42 $\mu$ ), ils sont moins nombreux : 14 .

(23) Grands crochets : $84 u$, petits $72 u$.

(24) Double couronne de 54 à 62 crochets ; grands : 86,$25 ; 93,75 ; 97,5 u$; petits : 67,$25 ; 67,50 ; 69,37 ; 71,25 ; 75$ u.

Fig. 65-67. - Anomotaenia, cf. trigonocephala (H. Krabbe 1869), intestin de Pica pica (L. 1758). Richelieu, 29-8-1943.

Largeur du scolex, 0,25-0,35 mm. ; longueur, 0,225-0,300. Ventouses inermes, diamètre $0,12-0,13$, parfois jusqu'à $0,175 \mathrm{~mm}$. Rostre à 20 crochets en double couronne. Chez un individu, les crochets sont longs de $34 u$, chez un autre de $40 u$; leur plus grande largeur (perpendiculaire abaissée du sommet de ra garde sur le dos du crochet), 7,5 $\mu$. En invagination, le rostre est long de 0,3, large de $0,1 \mathrm{~mm}$. ; en protraction, il est long de 0,175 , large de $0,87 \mathrm{~mm}$. Strobile long d'environ $40 \mathrm{~mm}$., avec une largeur maximum de $1 \mathrm{~mm}$., 150-200 proglottis chez les plus grands exemplaires. Pores génitaux irrégulièrement alternes, presque à l'angle antérieur du proglottis ; au quart antérieur chez les proglottis âgés, plus longs que larges. $36-42$ testicules (diamètre, $30-40 \mathrm{w}$, dans la moitié postérieure du proglottis, entre les canaux excréteurs longitudinaux. Poche du cirre petite, ovale, $50-65 \times 75-100 u$. Cirre pelotonné dans la poche. Canal déférent décrivant de nombreuses sinuosités dans le tiers poral de la largeur du proglottis, n'atteignant pas le bord antérieur du proglottis. Vagin dirigé obliquement, un peu postérieur aux sinuosités du canal déférent, renflé en un réceptacle séminal volumineux, sphérique ou ovoïde (diamètre, 75$125 \mathrm{u}$ ). Ovaire peu étendu, généralement au centre du proglottis ou un peu en arrière. Vitellogène peu développé. Utérus supposé sacciforme, non persistant, capsules ovifères, à un seul œuf. Oncosphère, 22-28 $\mu$ environ. Stéphane Deblock descripsit et delineavit.

Fig. 65, scolex ; fig. 66, crochets ; fig. 67, proglottis à maturité. Chez un autre individu de Pica pica (L.) ont été récoltés (4-8-1932) des spécimens d'un cestode appartenant vraisemblablement à la même espèce, mais avec 18 crochets de 32 et $35 \mu, 32-34$ testicules. 


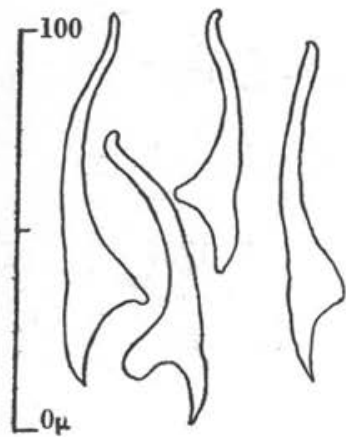

Fig. 68. - Dilepis undula (Schrank 1788). Intestin de Turdus ericetorum Turton 1807. Richelieu, 17-8-1936. R.-Ph. D. leg. 4 crochets d'un même scolex.

Dilepis undula (Schrank 1788) intestin Turdus merula (L. 1758) (25) (fig. 69)

Catenotaenia dendritica (Goe- intestin Sciurus vulgaris L. 1758 ze 1782) (26)

Gryporhynchus cheilancris- intestin Ardea cinerea L. 1758 trotus (K. Wedl 1855) (27) (fig. 70-72)

Paricterotaenia parina (F. Dujardin 1845) (28)

Paricterotaenia mesacantha

(C. R. Lopez-Neyra 1935)

(fig. 42)

Paruterina sp.

Liga gallinulae (P. J. Van Beneden 1858)

\section{Choanotaenia crateriformis} (Goeze 1782) (29) (fig. 73)

intestin Parus major L. 1758

duodénum

Oedicnemus oedicnemus 1758)

intestin Apus apus (L. 1758)

intestin Gallinula chloropus (L. 1758)

intestin Dryobates medius (L. 1758)

(25) 52 crochets en deux couronnes ; grands, 101-109u; petits, 78-83 u. Chez un autre exemplaire : 58 crochets, grands, $91-98$; petits, $80 \mathrm{u}$. Chez un troisième, les grands crochets sont longs de $103-108 u$, les petits de $83,7 u$.

(26) Les grands spécimens atteignent une longueur de $255 \mathrm{~mm}$. R.-Ph. D.

(27) Longueur, 37-40 mm.; largeur du scolex, 0,21 mm. ; largeur du rostre, $0,086 \mathrm{~mm}$. ; diamètre des ventouses, 0,147 . La première indication de proglottisation apparait à une distance de $0,76 \mathrm{~mm}$. de l'apex du rostre. A une longueur de $0.176-0,177$, les proglottis ne sont pas encore sexués. Un fragment pas encore sexué, long de 7,3 mm., comprend 46 proglottis plus longs que larges, déjà trapéziformes. Un fragment sexué, long de $8 \mathrm{~mm}$., comprend 35 proglottis longs de 0,23 en moyenne. La poche du cirre est longue de $70 \mu$ pour un proglottis sexué, trapézoïde, long de 0,23 , large antérieurement de 0,193 , postérieurement de 0,294 ; dans d'autres proglottis, la poche du cirre mesure $90 \times 40 \mu$. Les quatre testicules, ovales, mesurent $42 \times 60 \mu$. Les crochets atriaux sont longs d'environ 30 à 34,5 , avec une base large de 12 à $13,6 u$.

(28) Rostre à 20 crochets de $18,8 u$.

(29) Diamètre des ventouses, environ $200 u$. Crochets du rostre, 26 à $34 u$. Capsules ovigères, diamètre environ $37,5 u(30 \times 45 u)$; embryons hexacanthes, 18,75 à $22,5 u$. 
Choanotaenia musculosa (O. intestin Fuhrmann 1896) (30)

Choanotaenia guiarti (Shen Tseng 1932) var. scolopacis R.-Ph. D. nov. var. (fig. 4348)

Choanotaenia sp.

Anonchotaenia sp. (31)

Anonchotaenia sp. (31)

Dipylidium sp.

Biuterina sp.
Muscicapa (Ficedula) hypoleuca (Pallas 1764)

intestin Scolopax rusticola L. 1758

\section{Hymenolepididœe O. Fuhrmann 1907}

Hymenolepis sp. intestin intestin Certhia familiaris L. 1758

intestin Sitta europaea L. 1758

intestin Oriolus oriolus (L. 1758)

intestin Felis catus L. 1758 domest.

intestin Muscicapa striata (Pallas 1764)

Hymenolepis n. sp. (32) (fig. intestin 74)

Hymenolepis

J. G. Baer 1931

Crocidura mimula Gerrit Miller 1901

Crocidura russula (Hermann in Zimmermann 1780)

Neomys fodiens (Pallas 1756)

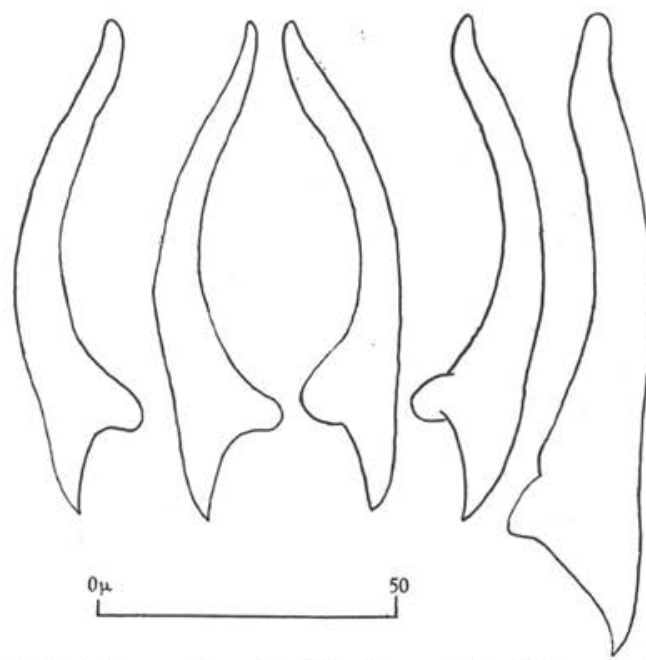

Fig. 69. - Crochets d'un scolex de Dilepis undula (Schrank 1788). Longueur : grands, 103-108 $\mu$; petits, 83,7 $\mu$. Richelieu, 1-8-1956.

(30) Capsules ovifères, $80 \mu$, avec un seul œuf (coque $40 \mu$, embryon $31 \mu$, crochets de l'onchosphère $17 \mathrm{u}$ ).

(31) Scolex inerme, diamètre des ventouses, $218 u$. Les proglottis du dernier cinquième de la longueur du strobile sont craspédotes. Les spécimens récoltés, longs de 15 à $30 \mathrm{~mm}$., sont immatures.

(32) Longueur, $6 \mathrm{~mm}$. ; largeur, 0,55. Tête grosse, avec un diamètre de 0,2 et une longueur de $0,31 \mathrm{~mm}$. Couronne simple de 26 crochets longs de $21_{\mathrm{u}}$ (base $16 \mu$, épaisseur $3 \mu$ ). Ventouses, diamètre 48-50 $\mu$. Les trois testicules en ligne droite. 

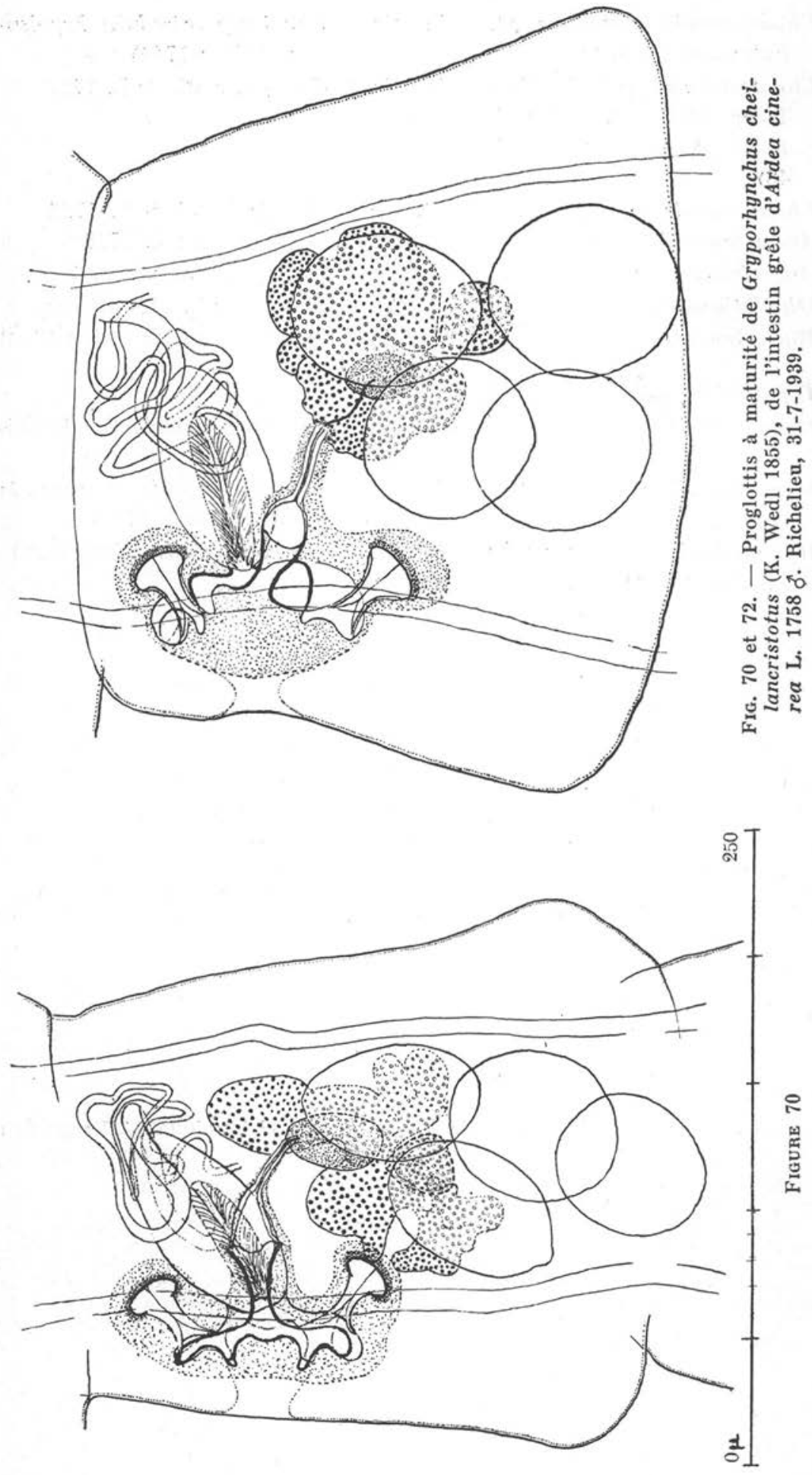
Fig. 71, crochets de l'atrium génital du même individu. R.-Ph. D. leg. et delin.

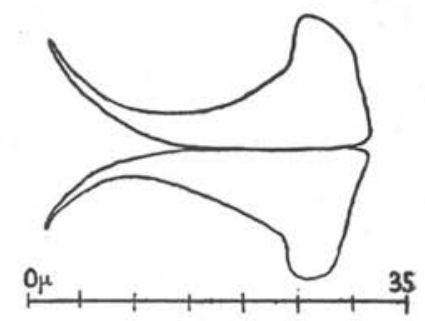

Hymenolepis scutigera (F. Du- intestin

Sorex araneus L. 1758 incl. $S$. jardin 1845) (33) (fig. 75-78) tetragonurus Hermann 1804

Hymenolepis cf. pistillum (F. intestin Talpa europaea L. 1758 Dujardin 1845) (34)

\section{Hymenolepis sp.}

intestin Eptesicus serotinus (Schreber 1775)

Hymenolepis grisea (P. J. Van intestin Myotis emarginatus (Et. GeofBeneden 1873) froy-St-Hilaire 1806)

Myotis myotis (Borkhausen 1797)

Hymenolepis sp. intestin Plecotus auritus (L. 1758)

Hymenolepis sp. intestin Rhinolophus ferrum-equinum (Schreber 1774)

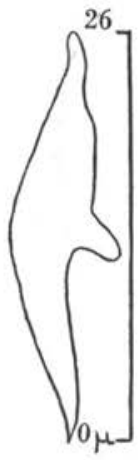

FIG. 73. - Crochet isolé du scolex de Choanotaenia crateriformis (Goeze 1782). Richelieu, 6-9-1950, R.-P. D leg.

(33) Crochets du rostre, 33 à $36 u$. Otufs, enveloppe externe 90-95 $\times$ 72-76,5 u ; enveloppe interne $36-42$ et $39-45 \mu$. Oncosphère $28 \times 33$ à $33 \times 38 u$. Crochets de l'embryon 12-13,5 u. Chez un autre individu de Sorex araneus L.: 10 crochets rostraux de $38,8 u$, ventouses, diamètre $100 u$; pores unilatéraux, pénis épineux.

(34) Longueur, 1-3 mm. Crochets rostraux très caducs, plus nombreux que 17 ; longueur, 12,726 $\mu$. Il y a seulement 13 à 17 proglottis distincts. La coque des œufs est ellipsoîdale et mesure en moyenne $64 \times 51 \mu$, avec une très grande variation, dimensions extrêmes : $54,99 \times 38,07 u$ et $80,37 \times 63,45 \mu$. Embryons hexacanthes, $39 \times 34 u$, en moyenne; dimensions extrêmes : $30,07 \times 33,84 u$ et $46,645 \times 44,415$ u. 


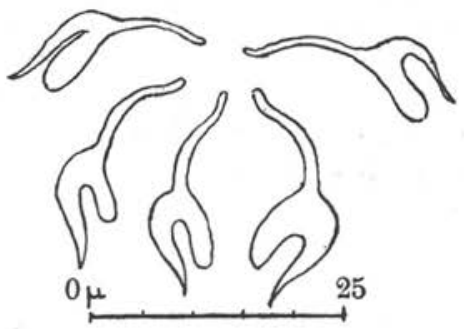

Fig. 74. - Hymenolepis n. sp., R.-Ph. D. de Crocidura russula (Hermann in Zimmermann 1790). Crochets. Les individus de ce Cestode, placés en eau physiologique, courbent en crosse leur extrémité antérieure, formant une boucle maintenue adhérente par une des ventouses du scolex. Richelieu, 15-9-1942. R.-Ph. D.

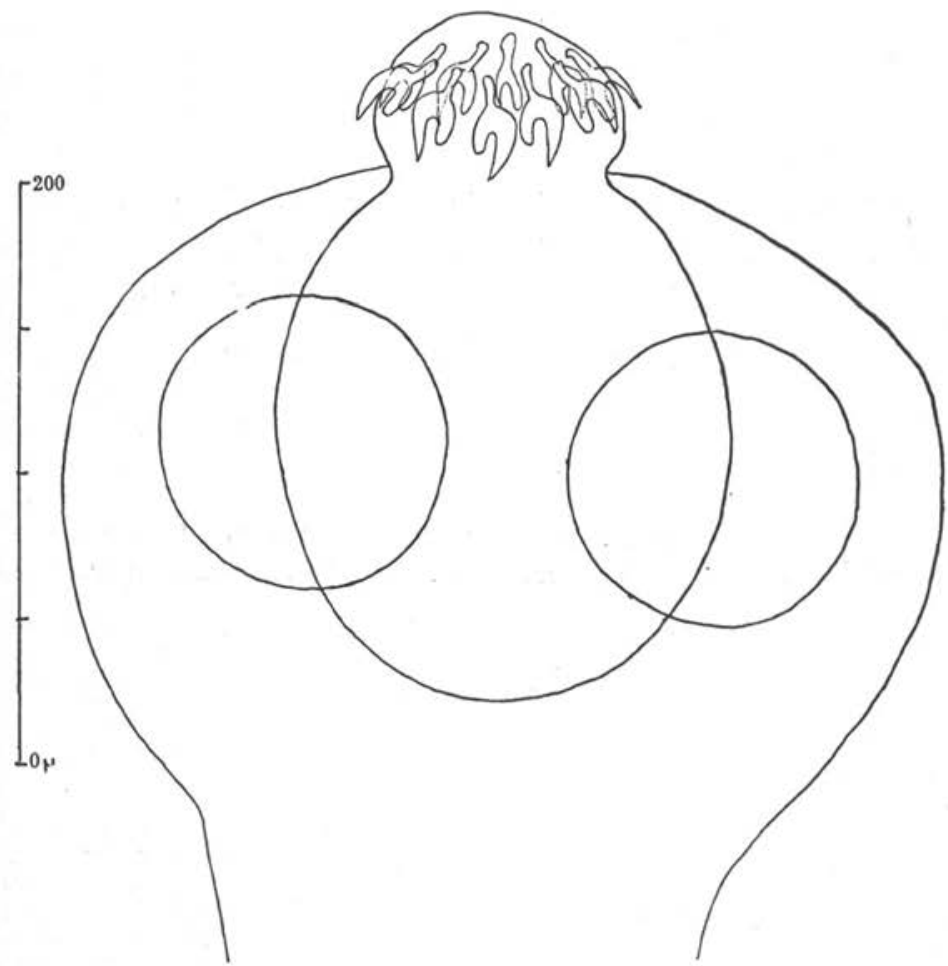

Fig. 75. - Hymenolepis ef. scutigera (F. Dujardin 1845). Intestin de Sorex araneus L. 1758, Richelieu, 12-9-1953. R.-Ph. D.

Scolex et ventouses. 


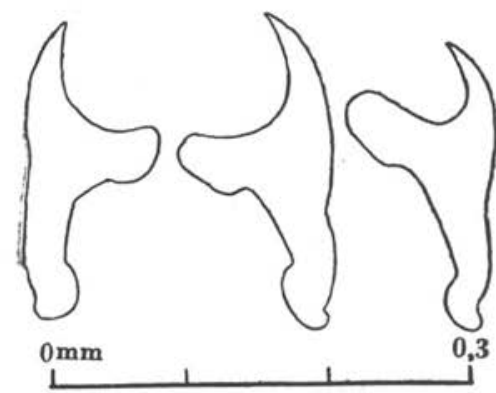

FIG. 76. - Hymenolepis sp. Intestin de Sorex tetragonurus Hermann 1804. Richelieu, 12-9-1942. R.-Ph. D. Trois crochets (longueur 33$36 \mu$ ).
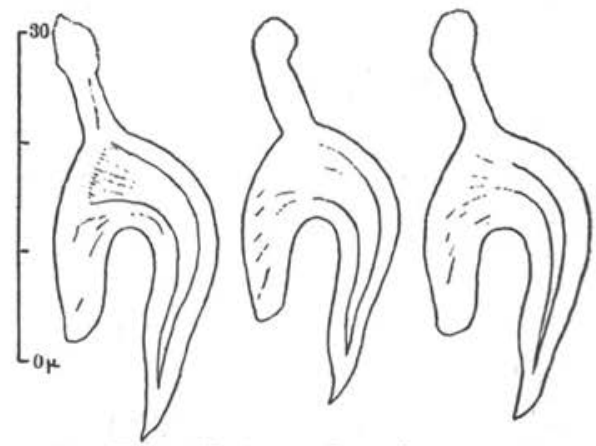

Fig. 77. - Trois crochets (longueur, $38,8 u$ ) d'Hymenolepis, cf. scutigera (F. Dujardin 1845), de Sorex araneus L., 1758, Richelieu, 12-91953. R.-Ph. D.

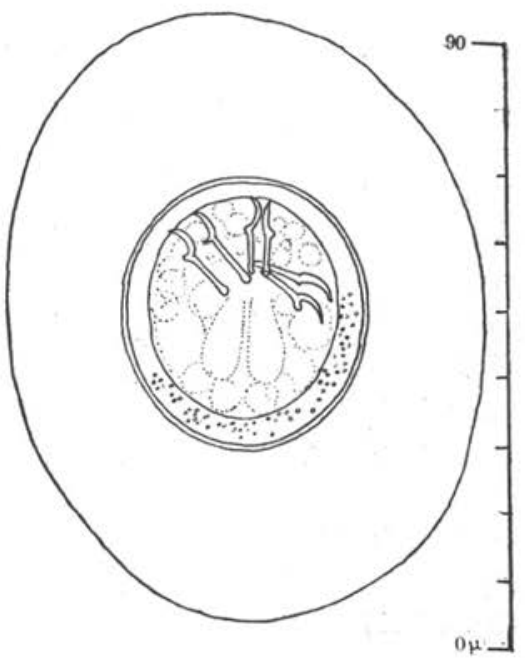

intestin Arvicola sapidus tenebricus Gerrit Miller 1903

intestin Eliomys quercinus (L. 1766) intestin Rattus (= Epimys) norvegicus (Berkenhout 1769)

intestin Rattus (= Epimys) norvegicus (Berkenhout 1769) 

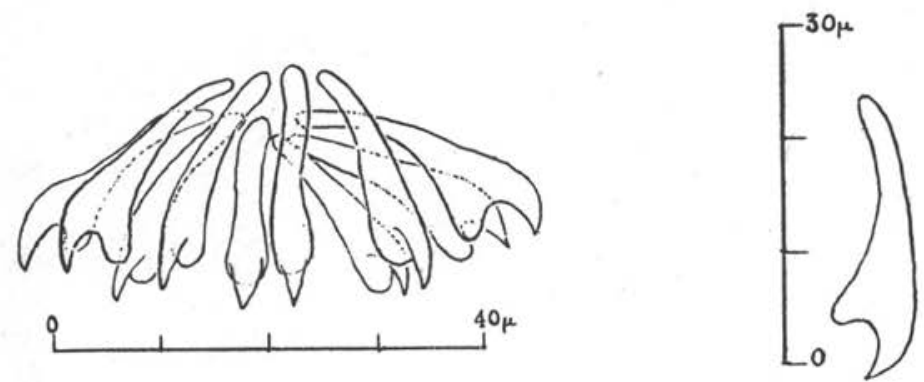

FIG. 79 et 80. - Hymenolepis (Weinlandia) serpentulus (Schrank 1788). Richelieu, 14-7-1932; R.-Ph. D. leg. Couronne de crochets et crochet isolé.

Hymenolepis parina (O. Fuhr- intestin Aegithalus caudatus (L. 1758) mann 1907)

Hymenolepis (Weinlandia) cf. intestin Turdus ericetorum Turton 1807 serpentulus (Schrank 1788)

Hymenolepis (Weinlandia) serintestin Anthus trivialis (L. 1758) pentulus (Schrank 1788) (36) (fig. 79-80)

Hymenolepis (Weinlandia) cf. intestin Pica pica (L. 1758) stylosa (Rud. 1809) (37)

Hymenolepis (Weinlandia) sp. intestin (38)

Hymenolepis sp. intestin Oriolus oriolus (L. 1758)

Hymenolepis (Weinlandia) sp. intestin Phylloscopus collybita (Vieillot. 1817)

Hymenolepis (Weinlandia) sp. intestin Turdus philomelos Brehm 1831 Hymenolepis (Weinlandia) sp. intestin Merula merula (L. 1758) (fig. 85-89)

(36) Un spécimen (14-7-1932) est long de $8,67 \mathrm{~mm}$., avec environ 110 proglottis. La plus grande largeur des proglottis est 0,25 , la plus grande longueur, 0,13 . Scolex : longueur, $215 u$; plus grande largeur, $195 u$. Ventouses : diamètre, $105 u$. Couronne de 10 crochets (fig. 79), longs de $25 \mu$ en moyenne (extrêmes 23 et $26 \omega$.

Un autre spécimen : longueur des crochets du rostre, $25 \mu$. Diamètre des ventouses, $81 u$. Longueur de la poche du cirre, $160 u$. Longueur des crochets de l'embryon, 27-29 $\mu$.

(37) 10 crochets de $28,9 u$.

(38) Scolex : diamètre, $88-90$ u. Ventouses : diamètre, 44-46 u. 10 crochets do 18-19 u. Diamètre des œufs, $43 \mathrm{u}$; diamètre de l'embryon hexacanthe, $34 u$; crochets de l'embryon, $13,79-14,5$ u. La forme des crochets diffère de celle de ceux d'H. clerci $O$. Fuhrmann 1924, qui a des ventouses beaucoup plus grandes. $(250 u)$ d'après Joyeux et Baer (1936, p. 213). 


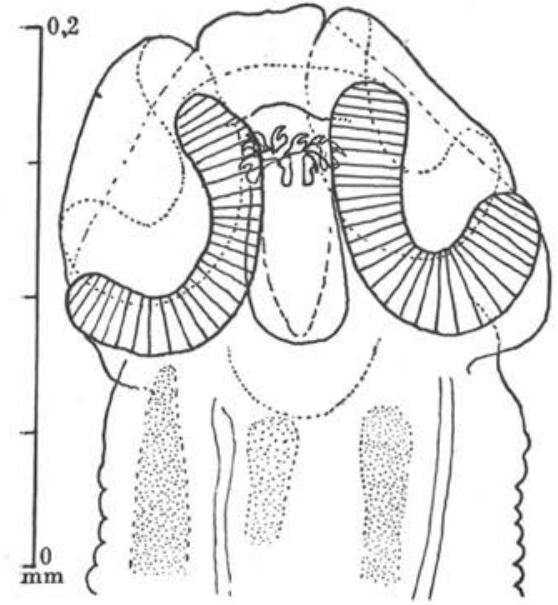

Figure 81

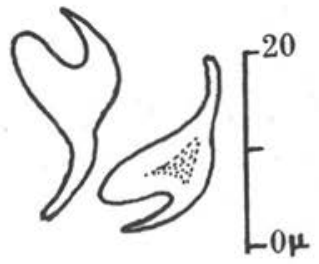

FIGURE 82

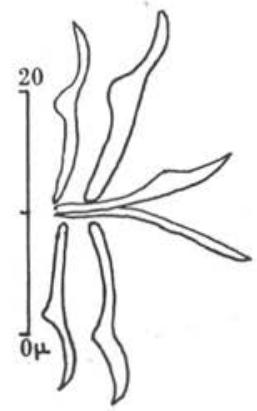

FIGURE 83

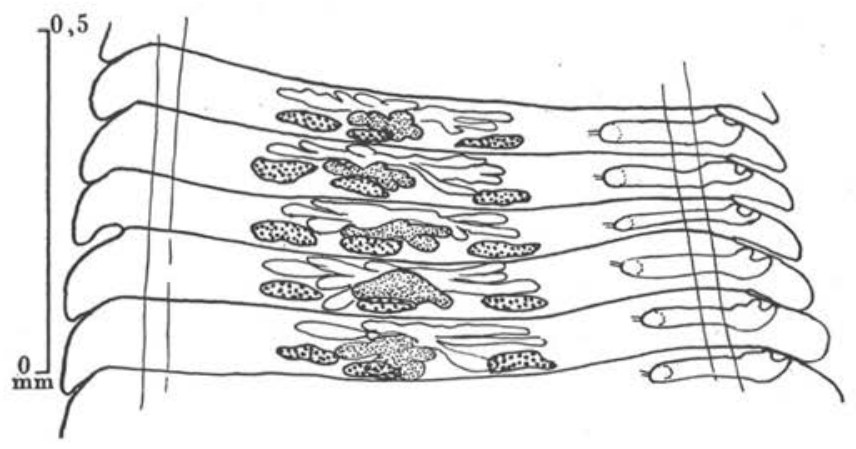

FIGURE 84

Fig. 81-84. - Hymenolepis (Hymenolepis) sp., de l'intestin de Sitta europaea L. 1758 ; Richelieu, 26-7-1947.

Fig. 81 , scolex ; fig. 82 , crochets du scolex ; fig. 83 , crochets de l'oncosphère ; fig. 84 , proglottis à maturité.

Il y a 10 crochets de $20 \times 11 \mu$, dont la forme est voisine de ceux de Weinlandia farciminosa (Goeze 1782), mais la disposition des glandes génitales n'est pas celle d'un Weinlandia.

Scolex : longueur, $0,14 \mathrm{~mm}$. ; largeur, $0,18 \mathrm{~mm}$; diamètre des ventouses, $0,105-0,110 \mathrm{~mm}$.; strobile long de $14 \mathrm{~mm}$. Poche du cirre longue d'environ $0,16 \mathrm{~mm}$. Crochets de l'oncosphère, long. $0,016 \mathrm{~mm}$. Stéphane Deblock delin. 


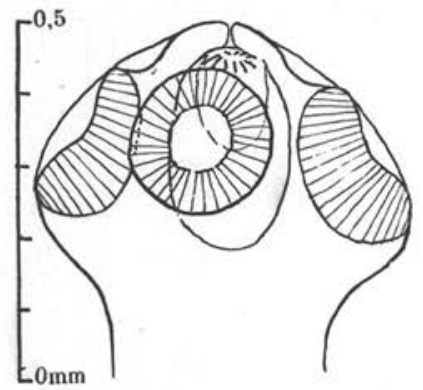

FigUre 85

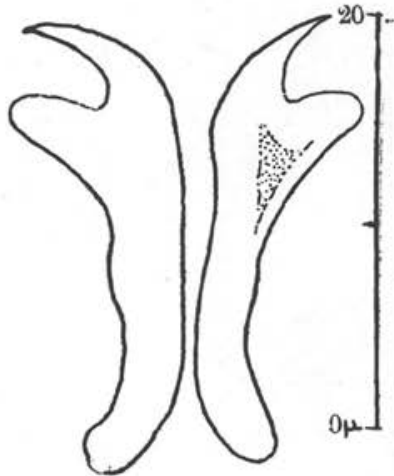

Figure 86

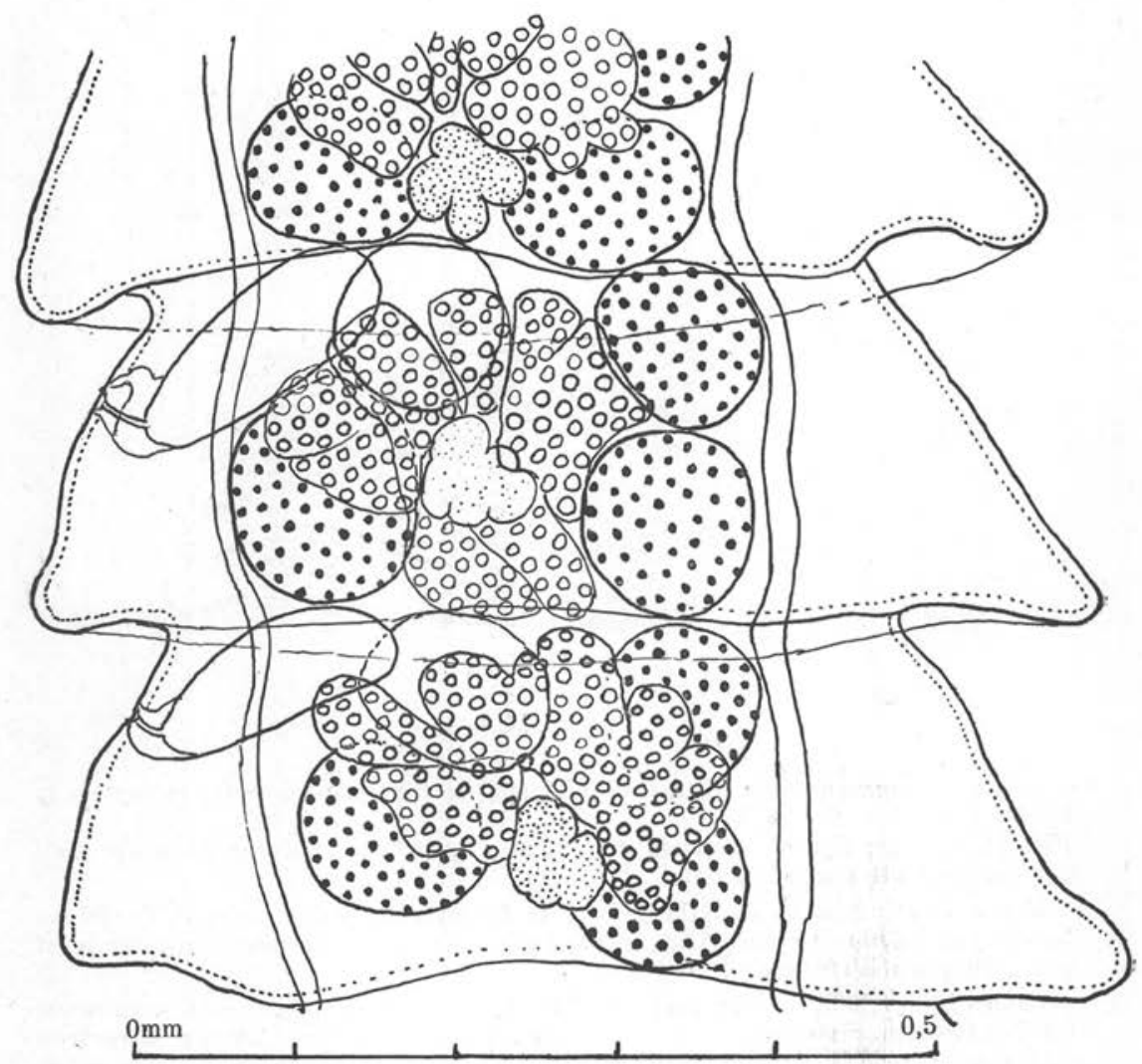

FIGURE 87 


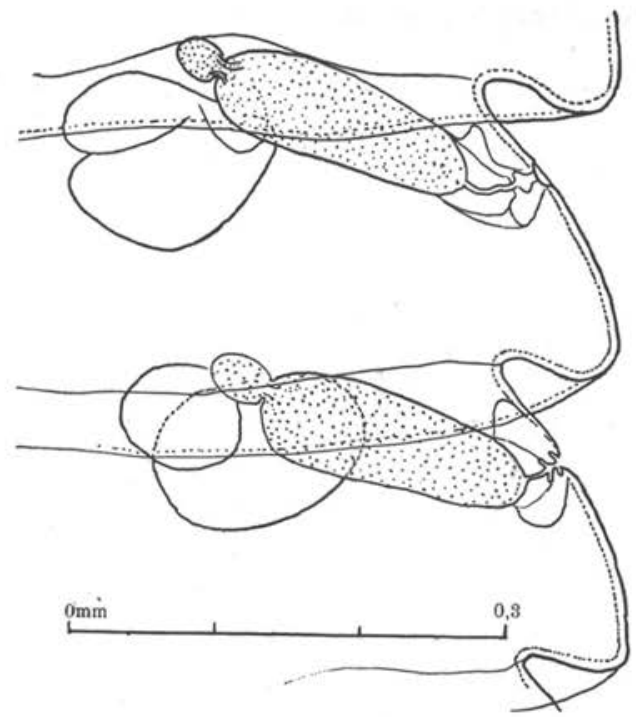

Figure 88

FIGURE 89

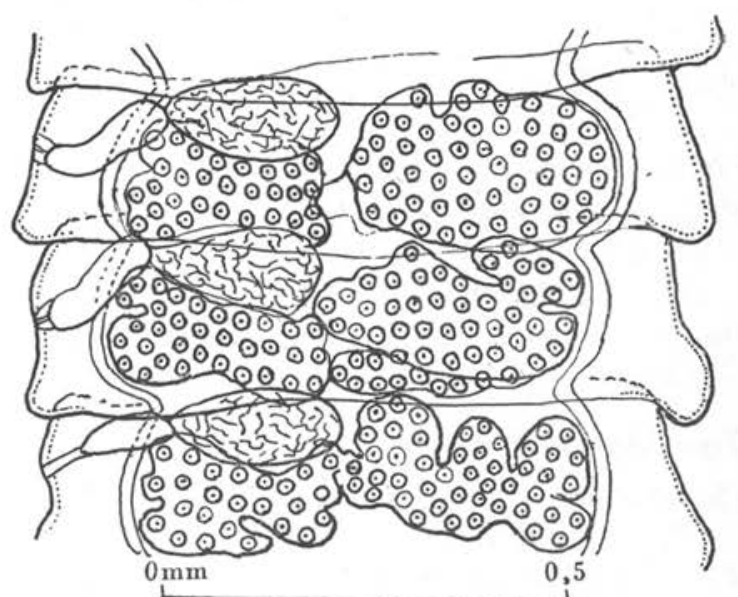

Fig. 85-89. - Weinlandia sp., de l'intestin de Merula merula (L. 1758), Richelieu, 19-8-1955.

Scolex long de 0,20 , large de $0,26 \mathrm{~mm}$. Ventouses, diamètre 0,110 à 0,125 . Dix crochets, $22 \times 7$ u. Strobile long d'environ $35 \mathrm{~mm}$. largeur maximum, 0,85 . Les proglottis à maturité ont une longueur moyenne de 0,25 et une largeur de 0,50 . Les trois testicules ont un diamètre moyen de $0,1 \mathrm{~mm}$. La poche du cirre mesure environ $0,25 \times 0,085$. La vésicule séminale semble présenter trois à cinq lobes ou diverticules dans quelques proglottis. Aucun proglottis n'est gravide.

Fig. 85, scolex : fig. 86, deux crochets vus sous des incidences un peu différentes ; fig. 87 . proglottis à maturité ; fig. 88 , poche du cirre et diverticules de la vésicule séminale ; fig. 89 , proglottis les plus âgés du spécimen étudié. Stéphane Deblock descrips. et delin.

Ce Weinlandia ressemble à serpentulus, mais la forme des crochets est complètement différente. 
Hymenolepis (Weinlandia) sp.

Hymenolepis (Weinlandia) cler-

ci O. Fuhrmann 1924 (40)

Hymenolepis (Weinlandia) naja

(F. Dujardin 1845)

Hymenolepis sp. (41)

Hymenolepis sp. (fig. 81-84)

Hymenolepis (Weinlandia) sp.

Hymenolepis sp. (42)

Hymenolepis (Weinlandia) sp.

Hymenolepis cf. columba (Zeder 1800)

Hymenolepis sp.

Hymenolepis (Hispaniolepis) villosa (M.-El. Bloch 1782)

Hymenolepis cf. multistriata (Rudolphi 1810) (43) (fig. 90-97)

Hymenolepis sp.

Fimbriaria sp.

Pseudhymenolepis cf. redonica Ch. Joyeux \& J. G. Baer 1935 (44) (fig. 4)

Diorchis ransomi L. N. Johri 1939

\section{Tœniida Ludwig 1886}

\section{Cladotaenia} cylindracea (M.-El. Bloch 1782)

Taenia saginata Goeze 1782

Coenurus serialis P. Gervais 1847 intestin

intestin

intestin

intestin

intestin

intestin

intestin

intestin

intestin

intestin

intestin

intestin

intestin

cavité

générale

intestin

intestin

cavité

générale

intestin

\section{Buteo buteo (L. 1758)}

Homo sapiens L. 1758

cavité

Erinaceus europaeus L. 1758

(39) Pour un proglottis long de 0,35 , large de $0,94 \mathrm{~mm}$., le diamètre du cirre épineux est d'environ $40 \mu$. Diamètre des $œ u f s, ~ 24 \times 30$ à $30 \times 36 u$. Crochets de l'oncosphère, $13,75 \mu$.

(40) 10 crochets de $20 \mu$. Utérus bilobé au début.

(41) Longueur des crochets du rostre, $17 \mu$; diamètre des ventouses, 60-63 $u$.

(42) 10 crochets de $60 \mu$. Poche du cirre de $117 \times 45$ à $203 \times 55 \mu$. Cirre épineux. Diamètre des œufs, $26 \mu$.

(43) 10 crochets de $50-52 \mu$.

(44) Proglottis isolés engagés dans les anfractuosités de la muqueuse, à laquelle ils sont adhérents par leur gros bout. Nous avons seulement trouvé des proglottis gravides (fig. 4). Capsules ovifères à un seul œuf, 43-49 $\mu$. Diamètre des cufs, $28.9 \times 34,6: 29 \times 30 ; 31,7$ à $37,5 \times 40,4 \mu$. Crochets de l'oncosphère, 14,4 à $15 \mathrm{u}$. 

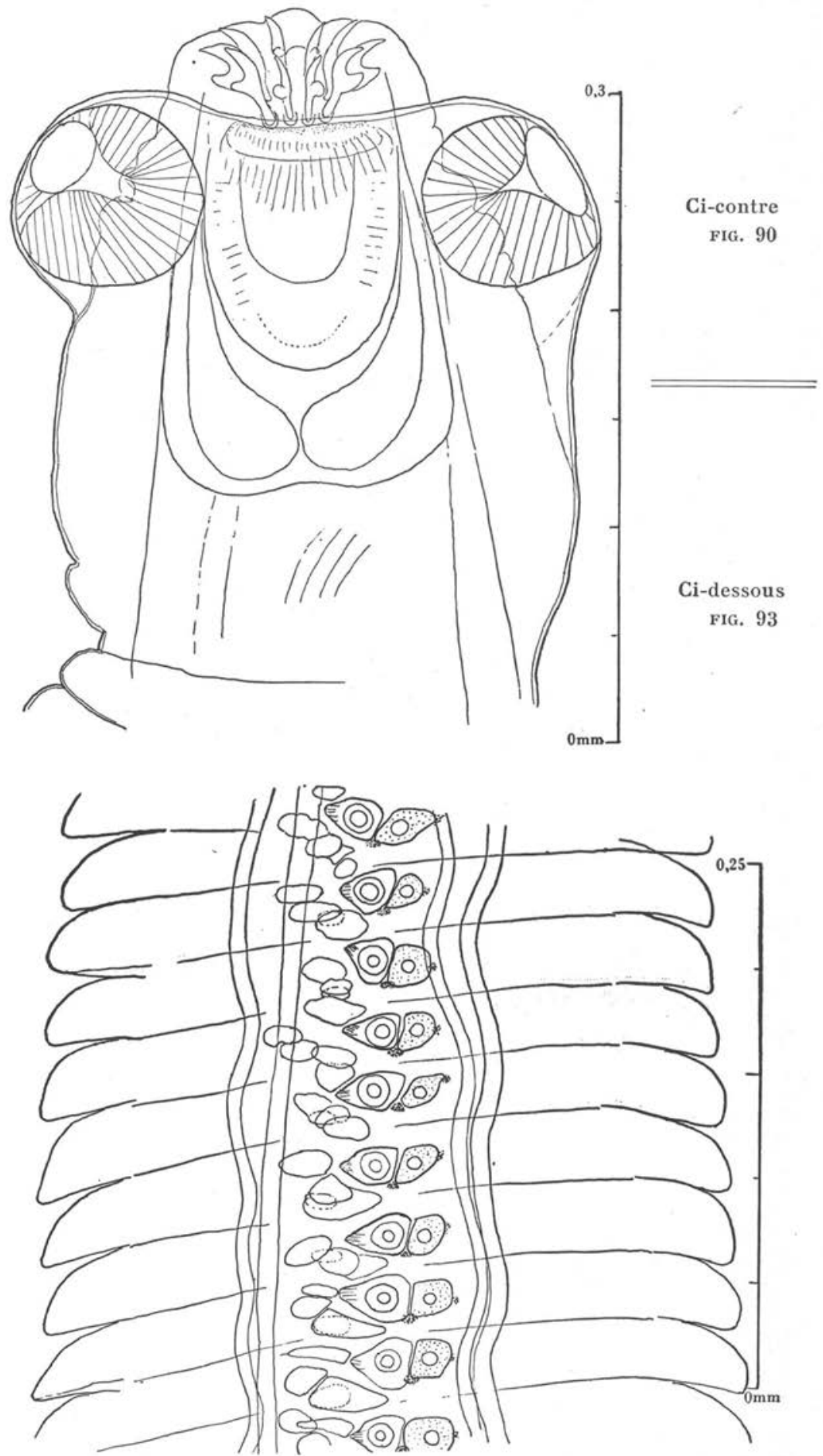

Ann. de Parasitologie, T. XXXVI, Nº 3, 1961. 


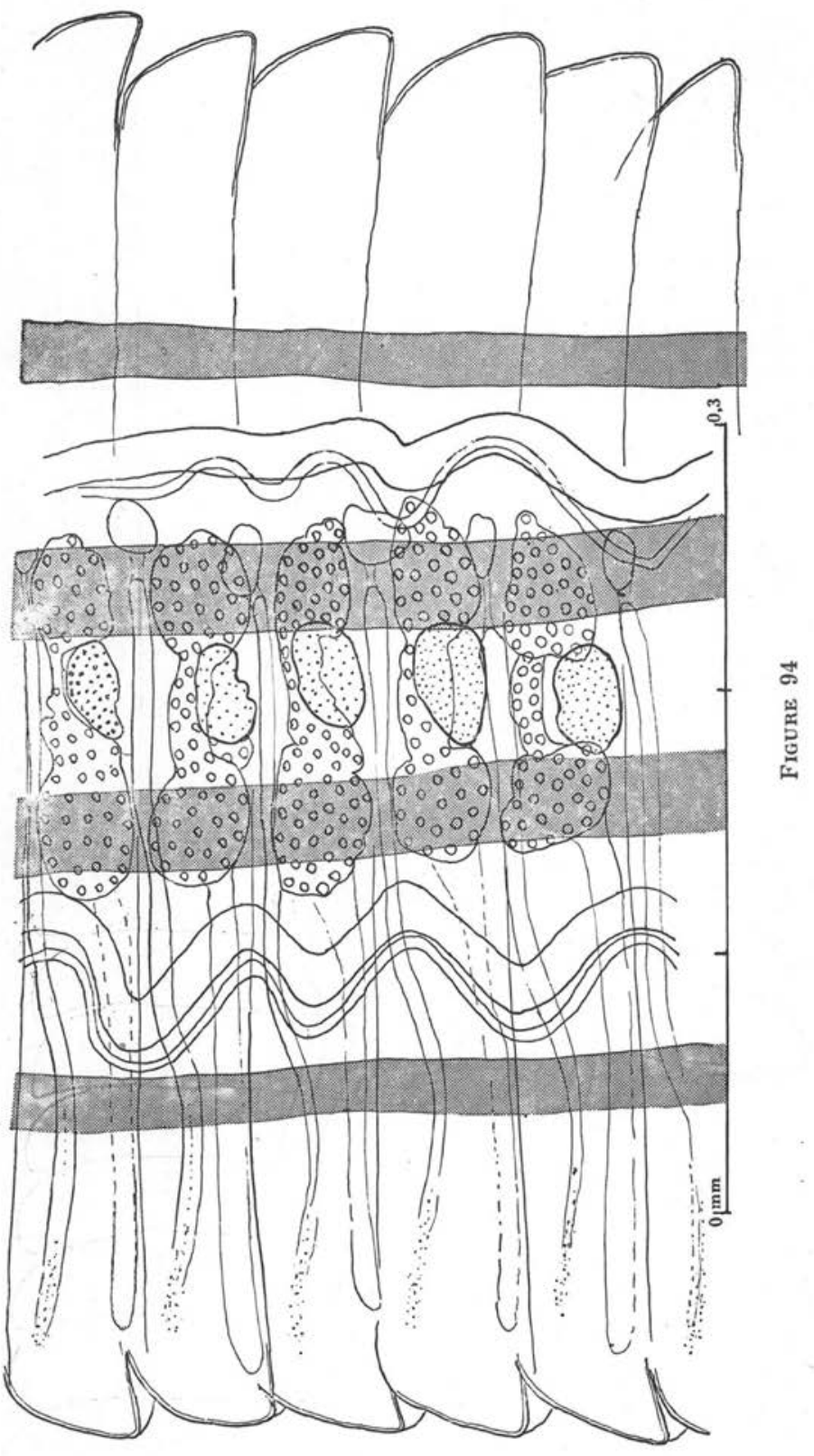


PARASITES : CESTODES

299
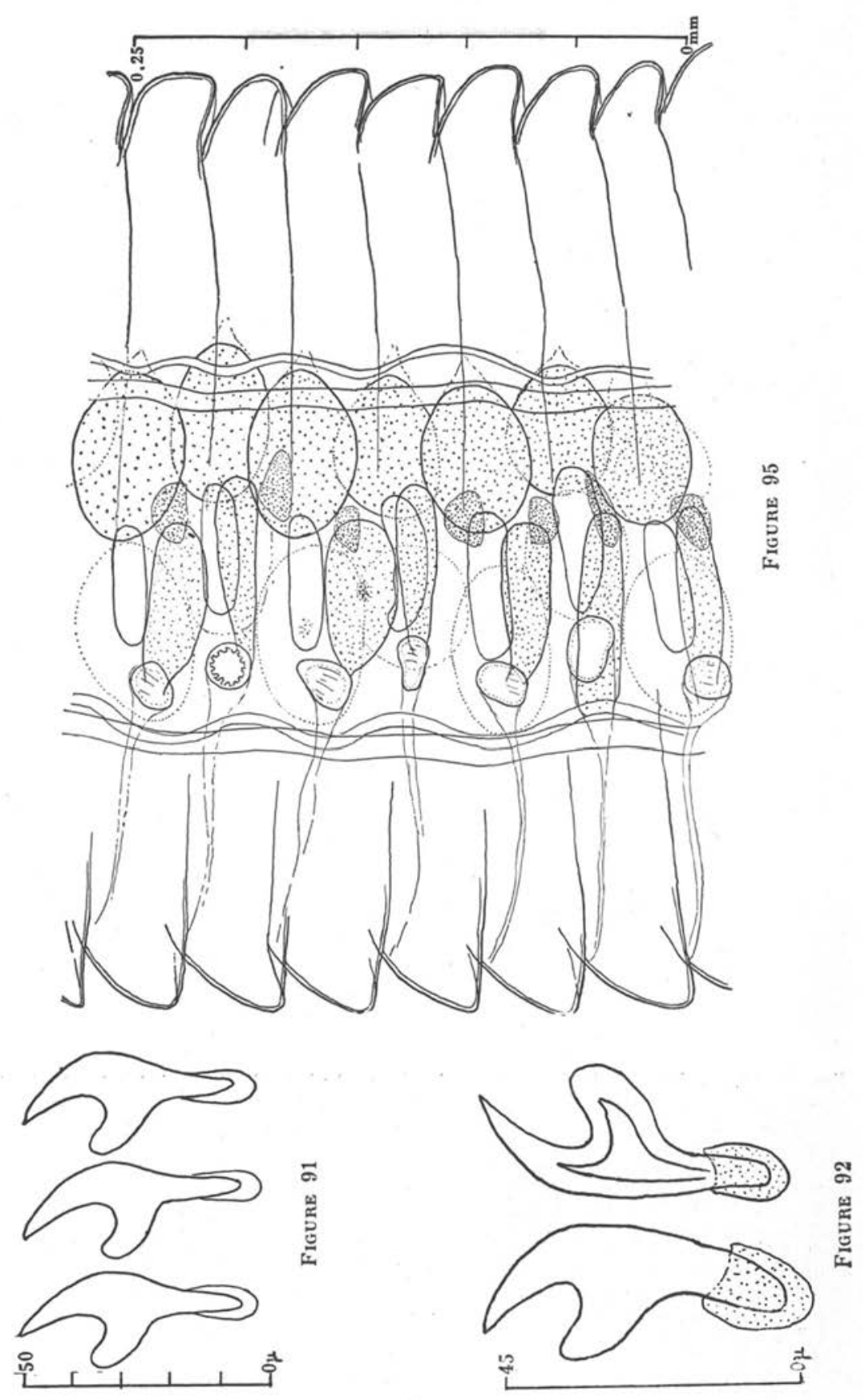


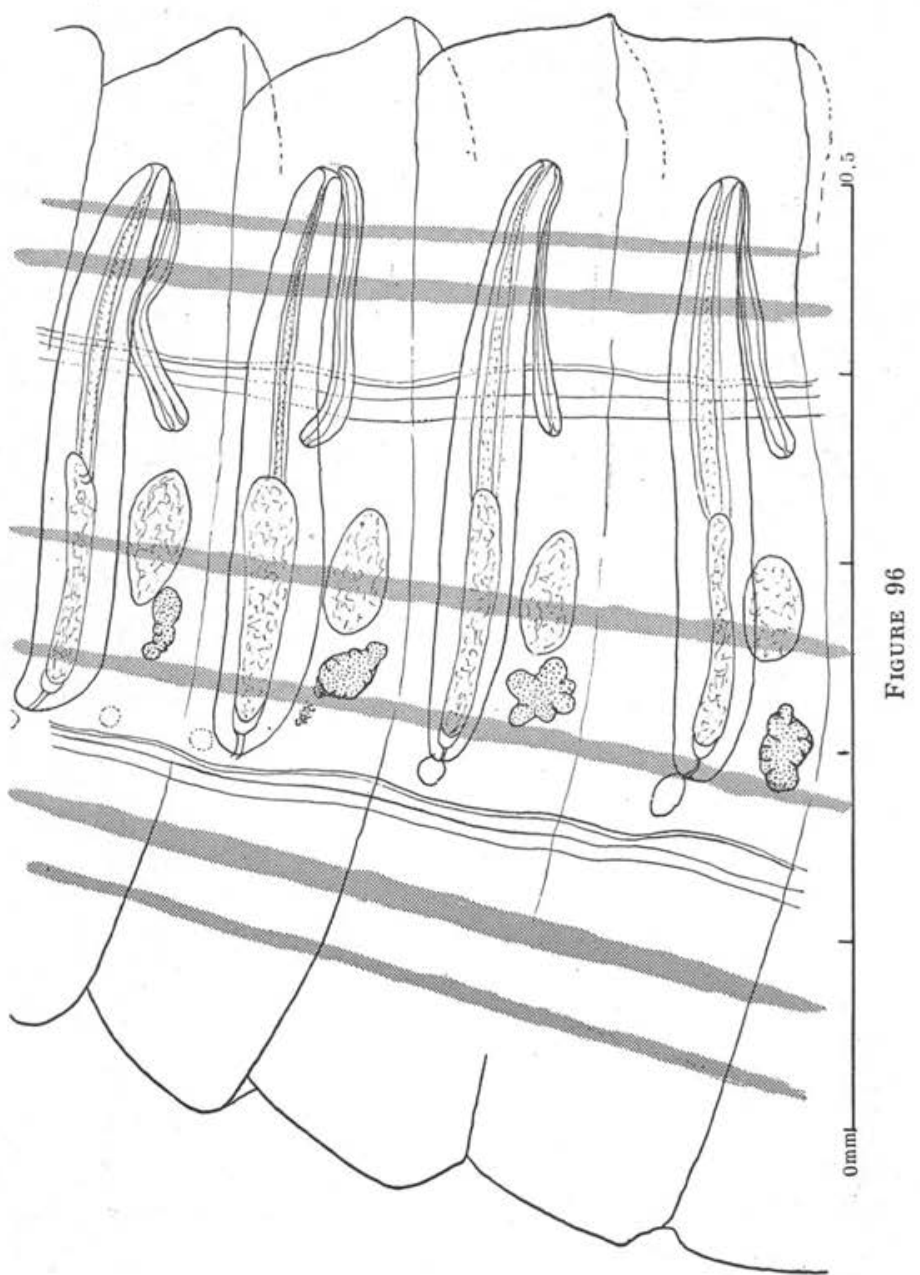

Fig. 90-97. - Hymenolepis, cf. multistriata (Rudolphi 1810), intestin de Podiceps ruficollis (Pallas 1764), Richelieu, 16-9-1955.

Fig. 90, scolex; fig. 91, crochets d'un autre scolex; fig. 92, crochets du scolex de la fig. 90 : fig. $93-97$, diverses parties du strobile. Ces figures ne sont pas une étude de l'anatomie, elles montrent seulement ce qui est observable sur des préparations colorées au carmin. Le côté poral est à droite sur les fig. 93, 96, 97 ; à gauche sur les fig. 94 et 95 .

Le scolex mesure environ $0,28 \times 0,28 \mathrm{~mm}$. : les ventouses ont un diamètre de 0,09 ; les crochets sont longs de 42 à 45 u et la base du manche est logée dans une sorte d'alvéole formant étui.

Le strobile a $20-24 \mathrm{~mm}$. de long, avec une largeur maximum de 0,64. Les proglottis sont excessivement nombreux. La poche du cirre mesure de $0,286-$ $0,296 \times 0,041-0,046$ à $0,338 \times 0,040-0,042 \mathrm{~mm}$. et passe ventralement aux deux canaux excréteurs longitudinaux. Le cirre est fortement épineux. La vésicule séminale externe devient très volumineuse. La fig. 91 a été dessinée par St. Deblock, les autres par R.-Ph. D. 

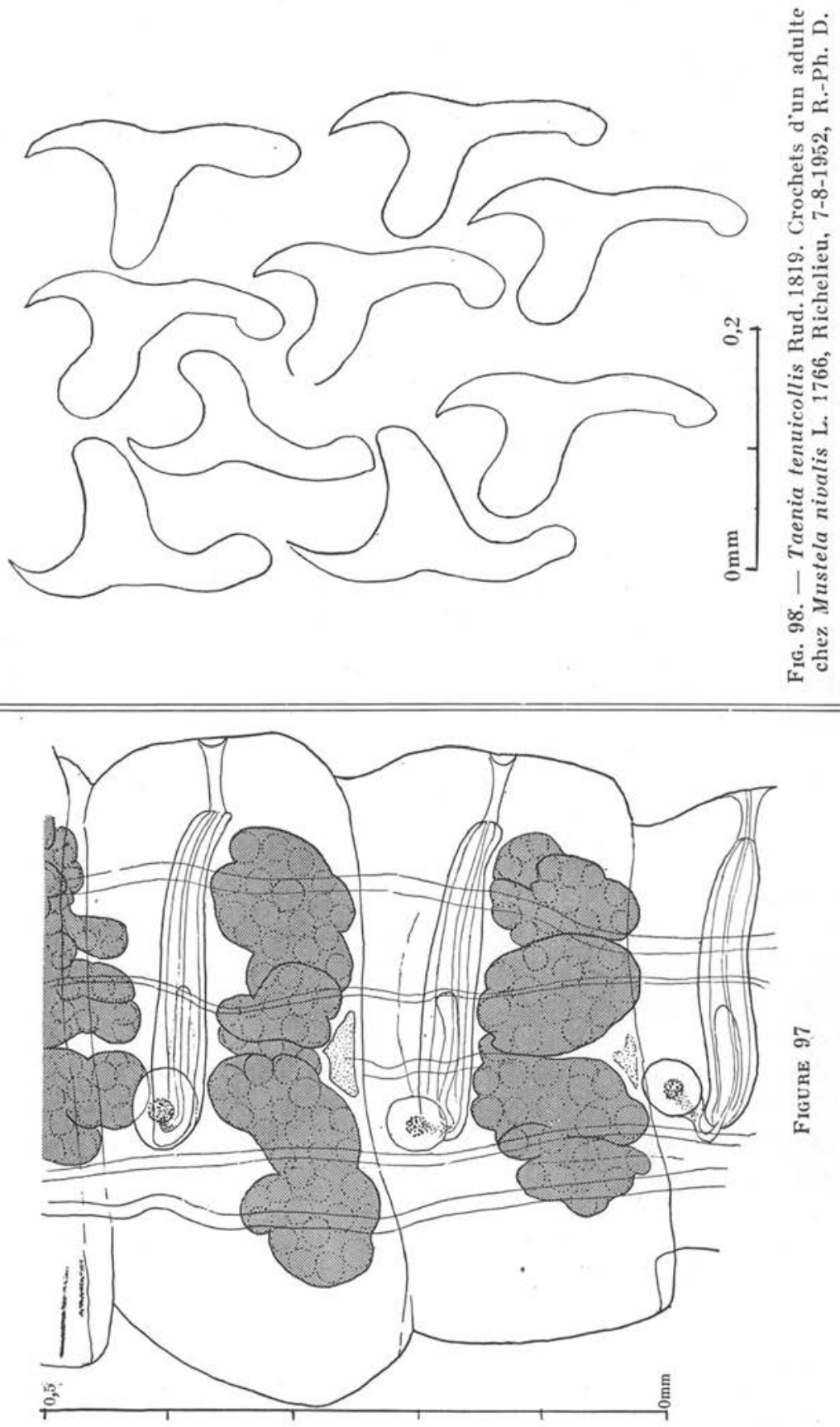
Oryctolagus cuniculus

(L. 1758)

Sciurus vulgaris L. 1758

Mustela nivalis L. 1766

Taenia tenuicollis (Rud. 1819) intestin (45) (fig. 98)

Taenia putorii (Zeder 1800) intestin Mustela putorius L. 1766 (46)

\section{Taenia sp.}

Taenia taeniaeformis (M.-El. intestin Bloch)

Cysticercus : Taenia taeniaeformis (M.-El. Bloch 1780) (= Cysticercus fasciolaris $\mathrm{Ru}$ dolphi 1808)

\section{foie Rattus (Epimys) norvegicus \\ (Berkenhout 1769) \\ foie $\quad$ Mus musculus L. 1758}

Fig. 99. - Cysticercus : Taenia tenuicollis Rud. 1819. Crochets d'un cystique du foie d'Evotomys glareolus (Schreber 1792), Richelieu, 30-8-1950, R.-Ph. D.

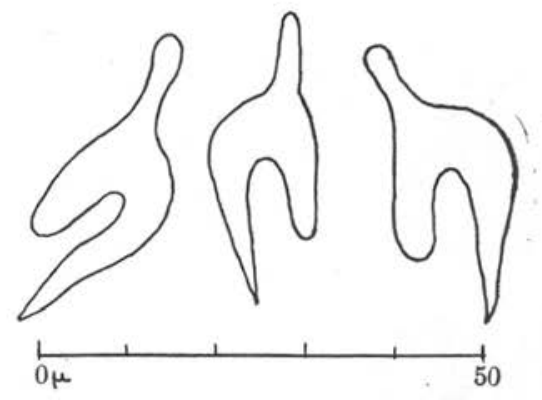

Cysticercus : Taenia tenuicollis Rudolphi 1819 (47) (fig. 99)

Taenia crassiceps Rudolphi 1810

Taenia (Multiceps) multiceps intestin Vulpes vulpes (L. 1758) (47 ${ }^{\mathrm{bis}}$ ) Leske 1780

Taenia pisiformis M.-El. Bloch intestin Vulpes vulpes (L. 1758) 1780 (48)

Cysticercus: Taenia pisiformis péritoine Oryctolagus cuniculus M.-El. Bloch 1780 foie

Evotomys (= Clethrionomys) glareolus (Schreber 1792)

intestin Vulpes vulpes (L. 1758)

(L. 1758)

(45) Grands crochets $23 \mu$, petits $21,3 u$. Chez un autre spécimen : grands croct ets $22,6 u$, petits $21 u$.

(46) Longueur, $230 \mathrm{~mm}$. Proglottis craspédotes. Rostellum à 38 crochets (1\%,8 à $13,6 u$ sur deux rangs. Ventouses, $144,8 \times 156,6 u$. OEufs, $21 \times 24 u$. Poche du cirre, $255 \times 115$ u. Utérus avec $13-16$ branches de chaque côté. Chez un autre spécimen, ventouses, $117 \times 141,117 \times 145,129 \times 157 \mathrm{u}$, ou environ $150 \mathrm{u}$ de diamètre.

(47) 52 crochets en deux couronnes; grands crochets, $23 \mu$; petits, 20,5-21 u. Diamètre des ventouses, $140-150 \mathrm{u}$.

$\left(47^{\text {bis }}\right)$ Récolté à Château-Garnier (Vienne).

(48) Grands crochets, $264 u$; petits, $143 \mu$. 


\section{NEMATODES}

APHASMIDIENS

MERMITHOIDEA

Mermithidae indéterm. chez des Orthoptères, Hémiptères, Diptères ENOPLIDA

\section{Trichurina}

TRICHUROIDEA

\section{Trichuridae}

\section{Trichurinae}

Trichuris leporis (Froelich 1789)

Trichuris muris (Schrank 1788)

Trichuris trichiura (Linné 1771)

Trichuris sp.

\section{Capillariinae}

Capillaria corvorum (Rudolphi 1819)

Capillaria erinacei (Rudolphi 1819)

Capillaria exigua (F. Dujardin 1845)

Capillaria? hepatica (Bancroft 1893)

Capillaria incrassata (Diesing 1851)

Capillaria muris-sylvatici (Diesing 1851)

Capillaria myoxi-nitelae (Diesing 1851)

Capillaria picorum (Rudolphi 1819)

Capillaria putorii (Rudolphi 1819)

Capillaria resecta (Dujardin 1845)

Capillaria soricicola (Nishigori 1924)

Capillaria splenaeca (Dujardin 1845)

Capillaria strigis (Froelich 1802)

Capillaria sp.
Oryctolagus cuniculus

Oryctolagus cuniculus domesticus

Apodemus sylvaticus

Arvicola amphibius (*)

Sus scrofa domesticus

Sciurus vulgaris

Coloeus monedula

Corvus corone

Erinaceus europaeus

Erinaceus europaeus

Sciurus vulgaris

Sorex araneus

Sorex minutus

Apodemus sylvaticus

Evotomys glareolus

Eliomys quercinus

Dryobates major

Mustela nivalis

Putorius putorius

Garrulus glandarius

Crocidura russula

Sorex araneus

Sorex minutus

Athene noctua

Tinca tinca

(*) Voir Arvicola sapidus tenebricus Gerrit Miller 1908. 
Capillaria sp.

Capillaria sp.

Capillaria sp.

Capillaria sp.

Capillaria sp.

Capillaria sp.

Capillaria sp.

Capillaria sp.

Capillaria sp.

Capillaria sp.

Capillaria sp.

Capillaria sp.

Capillaria sp.

Capillaria sp.

Capillaria sp.

Capillaria sp.

Capillaria sp.

Capillaria sp.

Capillaria sp.

Capillaria sp.

Capillaria sp.
Oedicnemus oedicnemus

Columba livia

Tyto alba

Buteo buteo

Strix aluco

Turdus viscivorus

Turdus philomelos

Pica pica

Emberiza cirlus

Perdix perdix

Sitta europaea

Anser anser domesticus

Phasianus colchicus

Gallus gallus domest.

Accipiter nisus

Erinaceus europaeus

Microtus arvalis

Putorius putorius

Oryctolagus cuniculus

Natrix natrix

Evotomys glareolus

\section{Trichosomoididae}

Trichosomoidinae.

Trichosomoides crassicauda (Bellingham Mus norvegicus 1840)

DIOCTOPHYMATINA

\section{Dioctophymatida}

Eustrongylides.

Eustrongylides sp. (larves)

Eustrongylides sp. (immatures)

Ardea cinerea

Podiceps ruficollis

\section{Soboliphymatidae}

Soboliphyme soricis Baylis \& Kung 1932 Sorex araneus

$$
\text { PHLYCTAENOPHORINA }
$$

\section{Robertdollfusidae}

Robertdollfusa paradoxa Chabaud \& Corvus corone Campana 1950 
RHABDITIDA

PHASMIDIENS

Rhabdi in :

RHABDITOIDEA

Rhabditidae

Rhabditis (sensu lato)

Mus musculus

Arvicola sapidus tenebricus

Apodemus sylvaticus

\section{Rhabdiasidae}

Rhabdias bufonis (Schrank 1788)

Bufo vulgaris

Rhabdias cf. entomelas (F. Dujardin 1845)

Anguis fragilis

Rhabdias sp.

Rana agilis

Rhabdias sp.

Natrix natrix

Strongylina

STRONGYLOIDEA

Strongyloidida

Strongyloides ratti (Diesing 1851)

Mus rattus

Strongyloides sp.

Mustela nivalis

Strongyloides sp.

Gallinula chloropus

Parastrongyloides winchesi (Morgan 1928) Talpa europaea

\section{Syngamidae}

\section{Syngaminae}

Syngamus trachea (Montagu 1811)

Corvus corone

Pica pica

Syngamus sp.

Turdus musicus

Syngamus sp.

Sturnus vulgaris

Syngamus sp.

Turdus merula

Syngamus sp.

Tyto alba

\section{Ancylostomatidae}

\section{Uncinariince}

Uncinaria stenocephala (Railliet 1884) Vulpes vulpes

Ancylostomatinae

Ancylostoma sp.

Felis catus

TRICHOSTRONGYLOIDEA

\section{Amidostomatidae}

Amidostomatinae 
Amidostomum anseris (Zeder 1800)

Anas boschas $\left({ }^{*}\right)$

Anser anser domest.

\section{Trichostrongylidae}

Molineinae

Oswaldocrusia cf. auricularia (Zeder Natrix natrix 1800)

Oswaldocruzia cf. dispar (Dujardin 1845) Anguis fragilis

Oswaldocruzia sp.

Rana agilis

Oswaldocruzia sp.

Rana esculenta

Oswaldocruzia sp.

Lacerta viridis

Oswaldocruzia sp.

Bufo vulgaris

Molineus patens (Dujardin 1845)

Mustela nivalis

Putorius putorius

\section{Trichostrongylinae}

Trichostrongylus retortaeformis (Zeder Sciurus vulgaris 1800)

Oryctolagus cuniculus

Oryctolagus cuniculus domesticus

Graphidium strigosum Dujardin 1845

Oryctolagus cuniculus

Oryctolagus cuniculus domesticus

\section{Viannaiinae}

Morganostrongylus talpae (Morgan 1928) Talpa europaea

Longistriata confusa Desportes \& Cha- Crocidura russula (Herbaud mann)

Longistriata didas Thomas 1953

Crocidura mimula Miller

Longistriata buttnerae A.-G. Chabaud Crocidura mimula 1961

\section{Heligmosomatidae}

Heligmosomatinae

Heligmosomum costellatum (Dujardin Microtus arvalis 1845)

(*) boschas = platyrhynchos. 
Heligmosomum minutum (Dujardin 1845) Evotomys glareolus nec Linstow

Heligmosomum polygyrum (Dujardin Arvicola amphibius 1845)

Heligmosomum skrjabini (Schulz 1926) Evotomys glareolus

Nematospiroides dubius Baylis $1926 \quad$ Mus musculus

Apodemus sylvaticus

METASTRONGYLOIDEA

Metastrongylidae

Skrjabingylinae

Crenosoma striatum (Zeder 1800) Erinaceus europaeus

Skrjabingylus nasicola Petrov $1927 \quad$ Mustela nivalis

Putorius putorius

Filaroidinae

Filaroides mustelarum (Rudolphi 1809) Mustela nivalis

Putorius putorius

COSMOCERCOIDEA

Cosmocercida

Cosmocercinae

Oxysomatium brevicaudatum (Zeder Bufo vulgaris 1800)

Oxysomatium sp.

Oxysomatium sp.

Anguis fragilis

Aplectana sp.

Rana agilis

Aplectana sp.

Bufo bufo

Anguis fragilis

\section{Kathlaniidae}

Kathlaniinae

Megalobatrachonema campanae (Cha- Triturus palmatus baud \& Golvan 1957)

\section{Atractidae}

Atractince

Atractis sp.

Bufo vulgaris 
SEURATOIDEA

\section{Seuratoida}

Seuratinae

Seuratum mucronatum (Rudolphi 1809) Plecotus auritus

\section{OXYUROIDEA}

\section{Oxyuridae}

Pharyngodoninae

Pharyngodon spinicauda (Dujardin 1845) Alytes obstetricans

Lacerta muralis

Parathelandros medinae (Calvente 1947) Lacerta muralis

Lacerta vivipara

Oxyurinae

Syphacia obvelata (Rudolphi 1802)

Passalurus ambiguus (Rudolphi 1819)

Oxyuris (s. l.) alata (Rudolphi 1819)

\section{ASCARIDIOIDEA}

\section{Anisakiidøen., sp. ?, larves}

Anisakiinae

Raphidascaris acus (Bloch 1779)

Raphidascaris sp.

Raphidascaris sp.

\section{Ascaridiidce}

\section{Toxocarinae}

Porrocaecum depressum (Zeder 1800) Accipiter gentilis
Apodemus sylvaticus

Arvicola amphibius

Evotomys glareolus

Microtus arvalis

Mus musculus

Oryctolagus cuniculus

Oryctolagus cuniculus domesticus

Microtus agrestis

Turdus sp.

Accipiter nisus

Esox lucius

Blicca bjoerkna

Leuciscus (Gardonus) rutilus

Leuciscus (Scardinius) erythrophthalmus

Anguilla anguilla 


Porrocaecum ensicaudatum (Zeder 1800)
Porrocaecum spirale (Rudolphi 1795)
Porrocaecum sp.
Porrocaecum larves

Incertae sedis : «Ascaris gracillima» v. Linstow 1890 (1)

\section{Ascaridiinae}

Toxocara mystax (Zeder 1800)

Toxocara canis (Werner 1782)

Ascaris lumbricoides Linnaeus 1758

Ascaris suum Goeze 1782

Ascaridiinae

Ascaridia galli (Schrank 1788)

HETERAKIDIOIDEA

\section{Heterakidiidae}

\section{Heterakidiinae}

Heterakis gallinae (Gmelin 1790)

Heterakis isolonche Linstow 1906

Heterakis sp.

Heterakis sp.

\section{Subuluridae}

\section{Subulurinae}

Subulura sp.

Subulura sp.

\section{SUBULUROIDEA}

Accipiter nisus

Strix aluco

Corvus corone

Pica pica

Turdus merula

Athene noctua

Strix aluco

Turdus viscivorus

Turdus philomelos

Accipiter nisus

Vipera aspis

Cobitis (Nemachilus) barbatula

Felis catus domesticus

Canis familiaris

Vulpes vulpes

Homo sapiens

Sus scrofa L., domest.

Gallus gallus domest.
Gallus gallus domest.

Anas boschas

Perdix perdix

Phasianus colchicus

Tetrax tetrax

Phasianus colchicus

Meleagris gallopavo

Caccabis rufa 


\section{SPIRURIDA}

Camallanina

\section{CAMALLANOIDEA}

\section{Camallanidae}

Camallaninae

Camallanus lacustris (Zoega 1776) Perca fluviatilis

Camallanus sp.

Leuciscus (Gardonus) rutilus

\section{DRACUNCULOIDEA}

\section{Philometridae}

Philometrinae

Philometra ovata (Zeder 1803)

Phoxinus phoxinus

Tinca tinca

Leuciscus (Gardonus) rutilus

Leuciscus (Scardinius)

erythrophthalmus

\section{Dracunculida}

Avioserpentinae

Avioserpens galliardi Chabaud \& Cam- Ardea cinerea pana 1949

Thelaziidae

Rhabdochona denudata (Dujardin 1845) Leuciscus (Scardinius) erythrophthalmus

Spirurna

\section{SPIRUROIDEA}

\section{Spiruridae}

Spirurinae

Mastophorus muris (Werner 1782)

Spirura talpae (Gmelin 1790)

Protospirura sp.

Gongylonematinae

Gongylonema problematicum Schulz 1926
Evotomys glareolus 1

Cetonia aurata, larves

Talpa europaea

Microtus arvalis Pallas

Blaps mortisaga, larves.

- Evotomys glareolus 


\section{Rictulariinae}

Rictularia proni Seurat 1915

Apodemus sylvaticus

Rictularia sp.

Sciurus vulgaris

Myotis emarginatus

\section{Habronematidae}

Habronematidae

Cyrnea (Procyrnea) leptoptera (Rudolphi Buteo buteo 1819)

Habronema sp.

Accipiter nisus

\section{Tetramerida}

Tetramerinae

Tetrameres sp.

Emberiza cirlus

Tetrameres sp.

Oriolus oriolus

Tetrameres sp.

Phylloscopus collybit.

Tetrameres sp.

Columba palumbus

Tetrameres sp.

Anas boschas

Tetrameres sp.

Strix aluco

Tetrameres (Tropidocerca) sp.

Pernis apivorus

Tetrameres (Tropidocerca) sp.

Gallinula chloropus

Tetrameres (Tropidocerca) sp.

Tringa ochropus

\section{Acuariida}

non identifiés

Tyto alba

Acuariinae

Acuaria anthuris (Rudolphi 1819)

Garrulus glandarius

Pica pica

Corvus corone

Acuaria parorioli Chabaud \& Petter 1961 Acuaria spiralis (Molin 1859)

Oriolus oriolus

Pica pica

Phasianus colchicus

Turdus merula

Acuaria galliardi Chabaud \& Petter 1961 Muscicapa sp.

Acuaria subula (Dujardin 1845)

Erythacus rubecula

Acuaria paragalliardi Chabaud \& Petter Muscicapa striata 1961

Acuaria dollfusi Chabaud \& Petter 1961 Anthus trivialis Acuaria sp.

Anas boschas 
Acuaria (Dispharynx) sp.

Cosmocephalus obvelatus (Creplin 1825)

Echinuria uncinata (Rudolphi 1819)

Synhimantus (S.) laticeps (Rud. 1819)

Synhimantus (S.) robertdollfusi Desportes 1947

Synhimantus sp.

Cheilospirura hamulosa (Diesing 1851)

Seuratinae

Streptocara crassicauda (Creplin 1829)

Streptocara sp.
Cuculus canorus

Larus ridibundus

Anas boschas

Buteo buteo

Tinnunculus tinnunculus Accipiter nisus

Buteo buteo

Tinnunculus tinnunculus Accipiter nisus

Gallus gallus domest.

Anas boschas

Gallinula chloropus

\section{PHYSALOPTEROIDEA}

Physalopteridae

Physalopteridae

Physaloptera apivori Desportes 1946

Pernis apivorus

Physaloptera cf. galinieri L.-G. Seurat

Physaloptera sp.

Tinnunculus tinnunculus

Physaloptera sp.

Accipiter nisus

Buteo buteo

\section{FILARIOIDEA}

\section{Desmidocercidae}

\section{Desmidocercinae}

Desmidocercella numidica Seurat $1920 \quad$ Ardea cinerea

\section{Diplotriaen'idae}

Diplotriaeninae

Diplotriaena obtusa (Rudolphi 1802)

Diplotriaena tridens (Molin 1858)

Diplotriaena sp.

\section{Onchocercidae}

Icosiellinae

Icosiella neglecta (Diesing 1851)

Forcipomyia (Lasiohelea) velox, larves dans la nature et expériment. Microfilaires obtenues expér. chez Sycorax silacea Curtis 1839 
Rana esculenta

Dirofilariinae

Pelecitus fulicae-atrae (Diesing 1861) Podiceps ruficollis

Onchocercinae

Litomosa filaria (V. Beneden 1873) Plecotus auritus

Myotis emarginatus

Myotis myotis

Ornithofilariinae

Ornithofilaria mavis (Leiper 1909) Turdus musicus

Pseudaproctella inornata Anderson 1956 Turdus musicus

\section{RHABDITIDA DE COLEOPTERES}

\section{Rhabditidae}

Rhabditis stammeri Völk larves dans Necrophorus vestigator

1950

le segment
génital

Herschel 1807

Necrophorus humator Goeze 1777

Necrophorus vespillo (L. 1758)

Rhabditis sp. 2 J. Théodo- larve à l'extérieur Necrophorus vespilloides ridès 1955

et dans le

Herbst 1784

segment génital

Rhabditis insectivora Körner 1954

cavité générale, cavité sous-

élytrale et

surface externe

\section{Diplogastridœe}

Diplogaster hirschmannae

H. Sachs

segment génital

Geotrupes stercorarius (L. 1758)

G. mentator Marsh 1802

G. stercorosus (Scriba 1791)

G. vernalis (L. 1758)

Diplogaster sp.

cavité générale Necrophorus vespilloides et segment génital Herbst 1784 
cavité générale Dorcus parallelipipedus (L. et gaine du pénis 1767)

segment génital Typhaeus typhaeus

(L. 1758)

Geotrupes mutator Marsh 1802

G. spiniger Marsh 1802

G. stercorosus (Scriba 1791)

Rhabditida dont le genre segment génital n'a pas été identifié

Necrophorus interruptus Steph. 1803

$\begin{array}{cc}\text { segment génital } & \text { Necrophorus vespillo (L. } \\ \text { et cavité générale } & 1758 \text { ) } \\ \text { cavité générale } & \text { Necrophorus humator Goe- } \\ & \text { ze } 1777 \\ & \text { Ontholestes murinus } \\ & \text { (L. 1758) } \\ \text { gaine du pénis } & \text { Dorcus parallelipipedus (L. } \\ \text { et segment génital } & \text { 1767) }\end{array}$

\section{Oxyuridce}

Thelastoma macramphidum J. R. Christie 1931 var. gallica J. Théodoridès 1955

Thelastoma galliardi R.-Ph. Dollfus 1925

Cetonia aurata (L. 1758), larve

Cylindrojulus londinensis (Leach 1817)

Glomeris marginata Villers

SPIRUROIDEA

Larves non identifiées chez Glomeris marginata Villers

\section{ACANTHOCEPHALES}

EOACANTHOCEPHALA H. J. Van Cleave 1936

Neoechinorhynchidœ H. B. Ward 1918

Neojechinorhynchus rutili intestin Abramis brama (L. 1758)

(O. F. Müller 1780)

Phoxinus phoxinus

(L. 1758) 


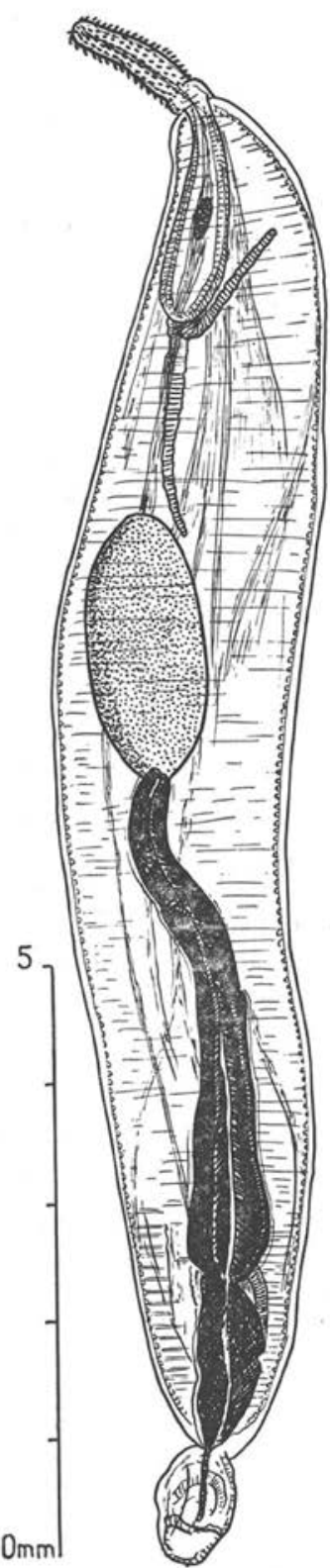

Figure 100

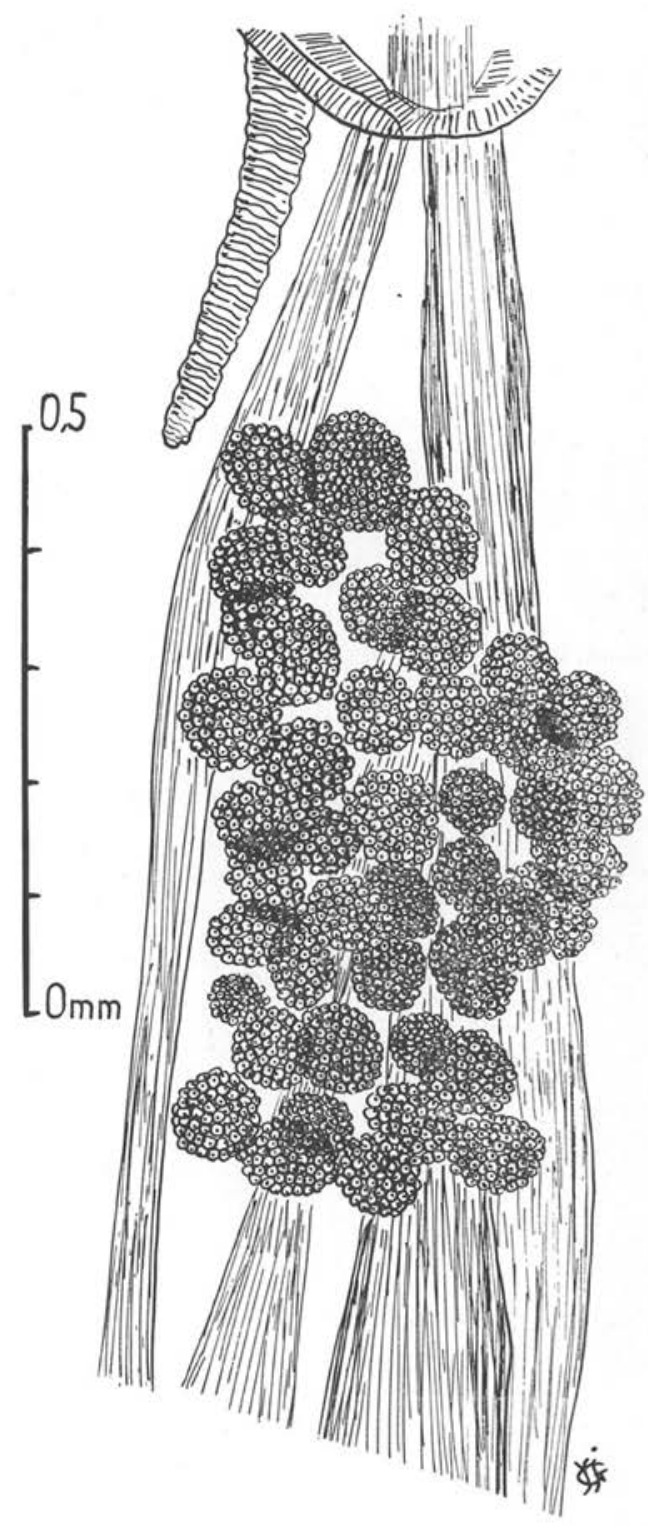

FIGURe 105

FIG. 100-105. - Prosthorhynchus cylindraceus (Goeze 1782), spécimens récoltés à Richelieu. Y. Golvan delin. 


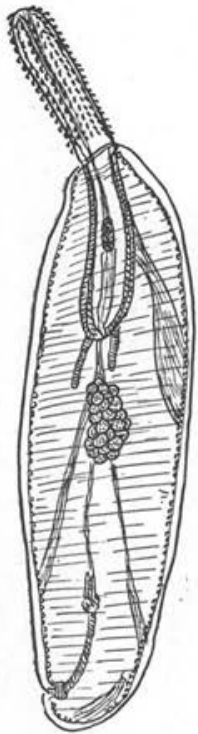

FIgure 102

Figure 101

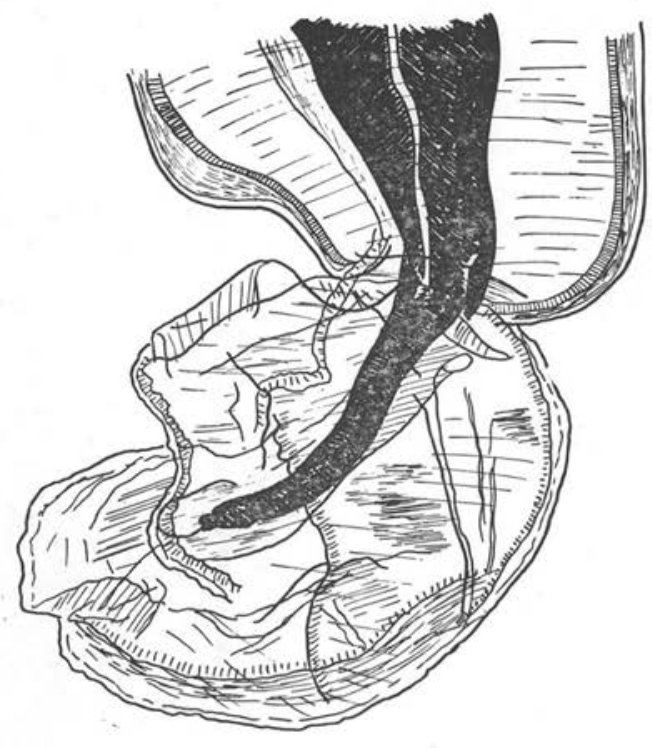

FigURE 103

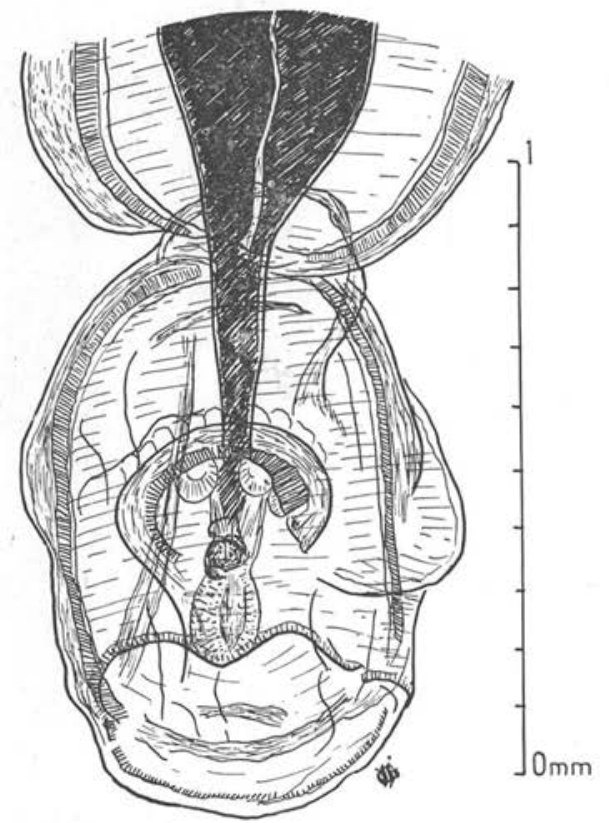

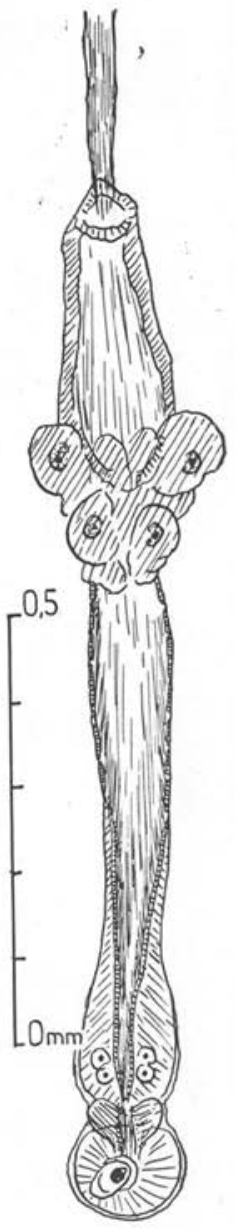

FigURe 104

Fig. 100, individu monorchide jeune, de l'intestin d'Erinaceus europaeus L. ; fig. 101, bourse caudale du même; fig. 102, \& juvénile libre dans l'intestin d'Erinaceus europaeus L.; fig. 103, bourse caudale en vue latérale d'un ${ }^{*}$ adulte de l'intestin de Turdus merula L. ; fig. 104, appareil utéro-vaginal d'une $\&$ adulte de l'intestin de Turdus merula L. ; fig. 105 , ovaire folliculaire fragmenté chez le juvénile de la fig. 102, libre dans l'intestin d'Erinaceus europaeus L. 1758. Y. Golvan delin. 

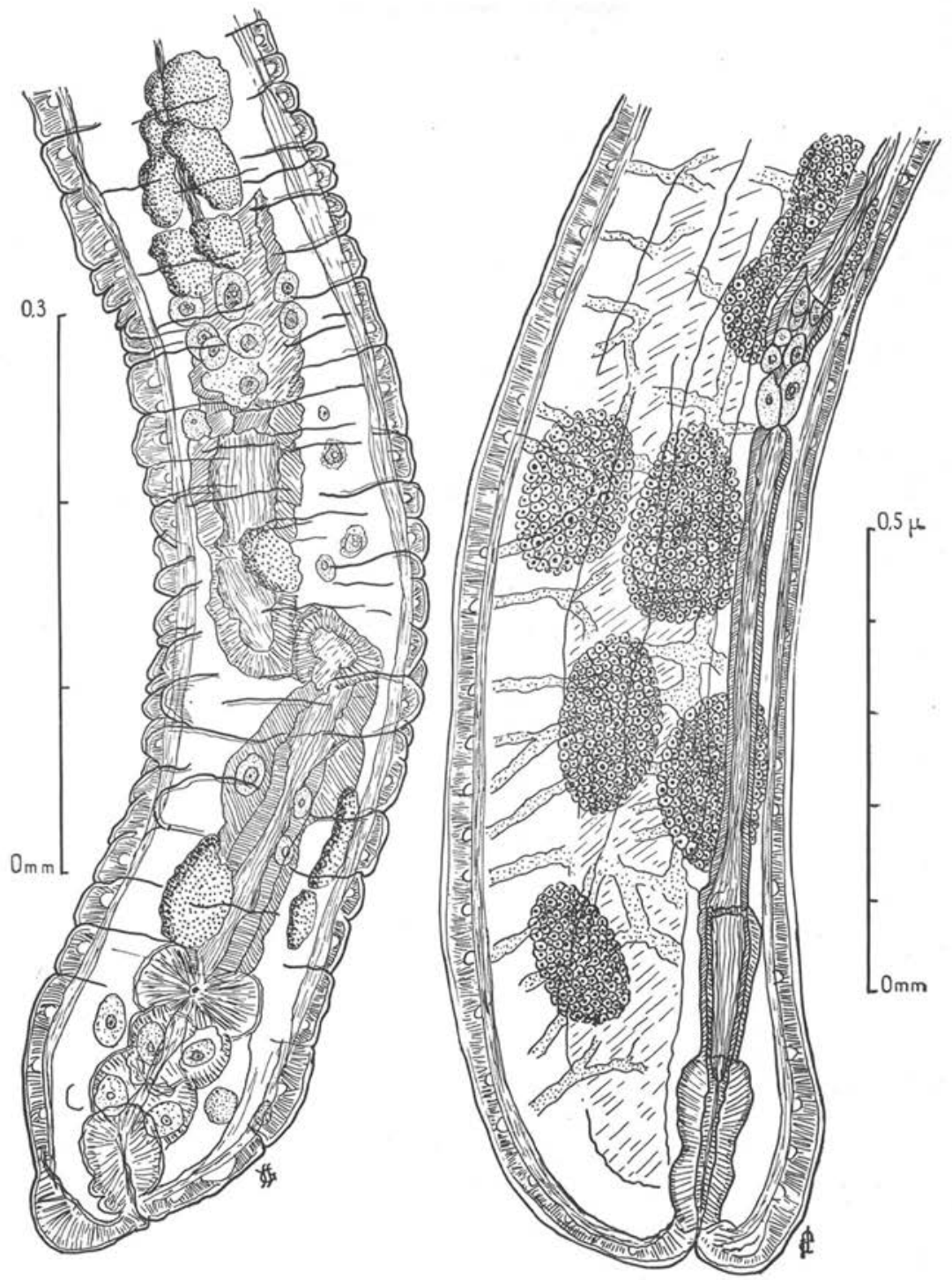

Fig. 106 (à gauche). - Appareil utéro-vaginal d'une $q$ juvénile de Centrorhynchus buteonis (Schrank 1788), de Buteo buteo (L. 1758), $\mathrm{D}^{\mathrm{r}}$ Yves Golvan delin., Salins-de-Badon, 12-4-1958. Y. Golvan delin.

Fıg. 107 (à droite). - Appareil utéro-vaginal d'une $\subsetneq$ juvénile de Centrorhynchus aluconis (O. F. Müller 1780), de Strix aluco L. 1758, Dr Yves Golvan delin.; Richelieu. Y. Golvan delin. 


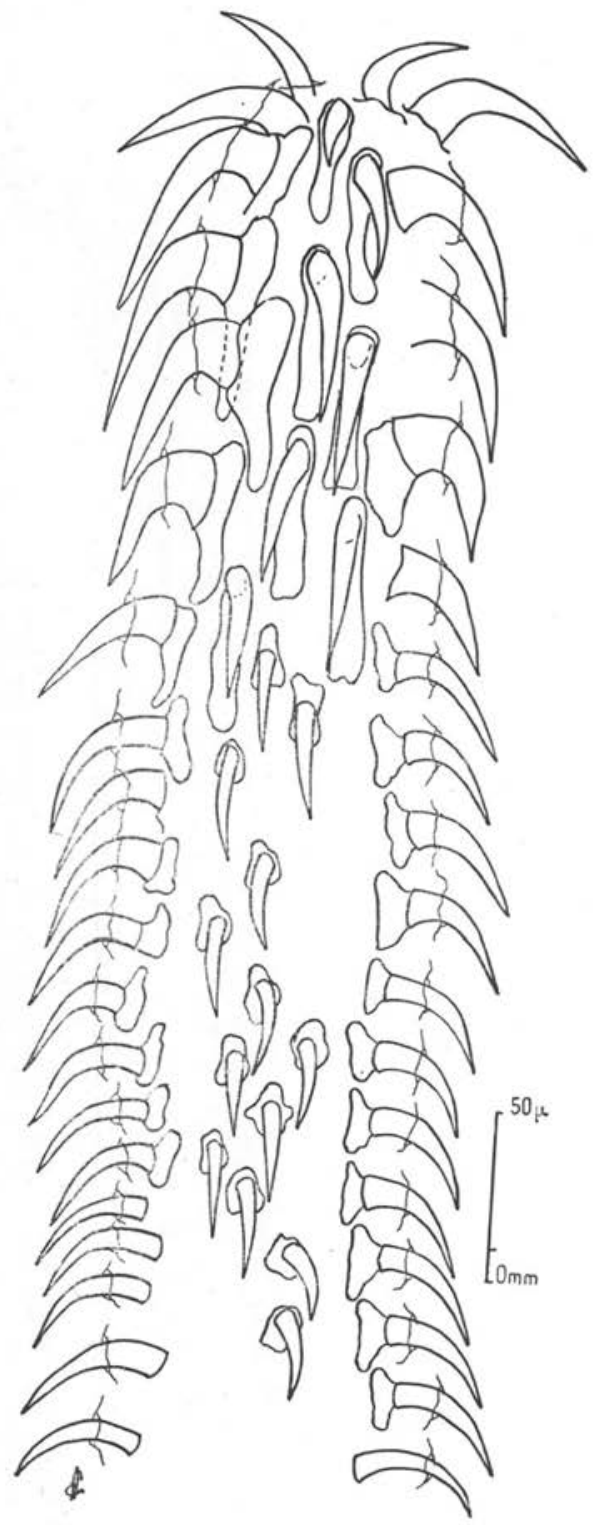

Figure 109

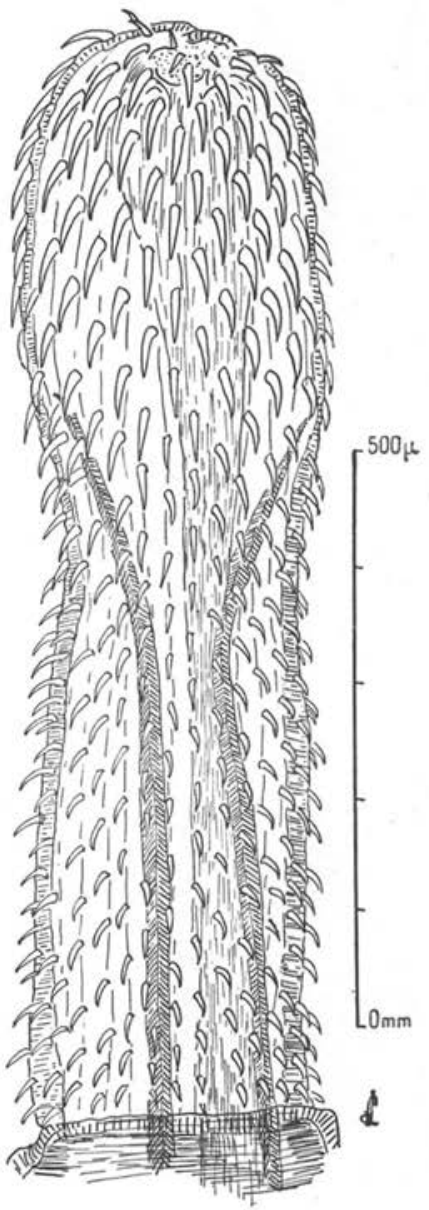

FIGURE 108

FiG. 108 et 109. - Centrorhynchus buteonis (Schrank 1788) de l'intestin de Tinnunculus tinnunculus (L. 1758), Salins-de-Badon (Bouches-du-Rhône), 12-1-1958. Rostre, 7-10 files de crochets; 9 à 14 files d'épines, Yves Golvan delin.

La présence à Richelieu de C. buteonis (Schrank) est incertaine, les spécimens récoltés ayant été accidentellement détruits, mais nous figurons le rostre (jusqu'à présent non figuré) d'un spécimen de cette espèce, pour permettre de la reconnaitre si elle vient à être trouvée à Richelieu. 
Figure 110
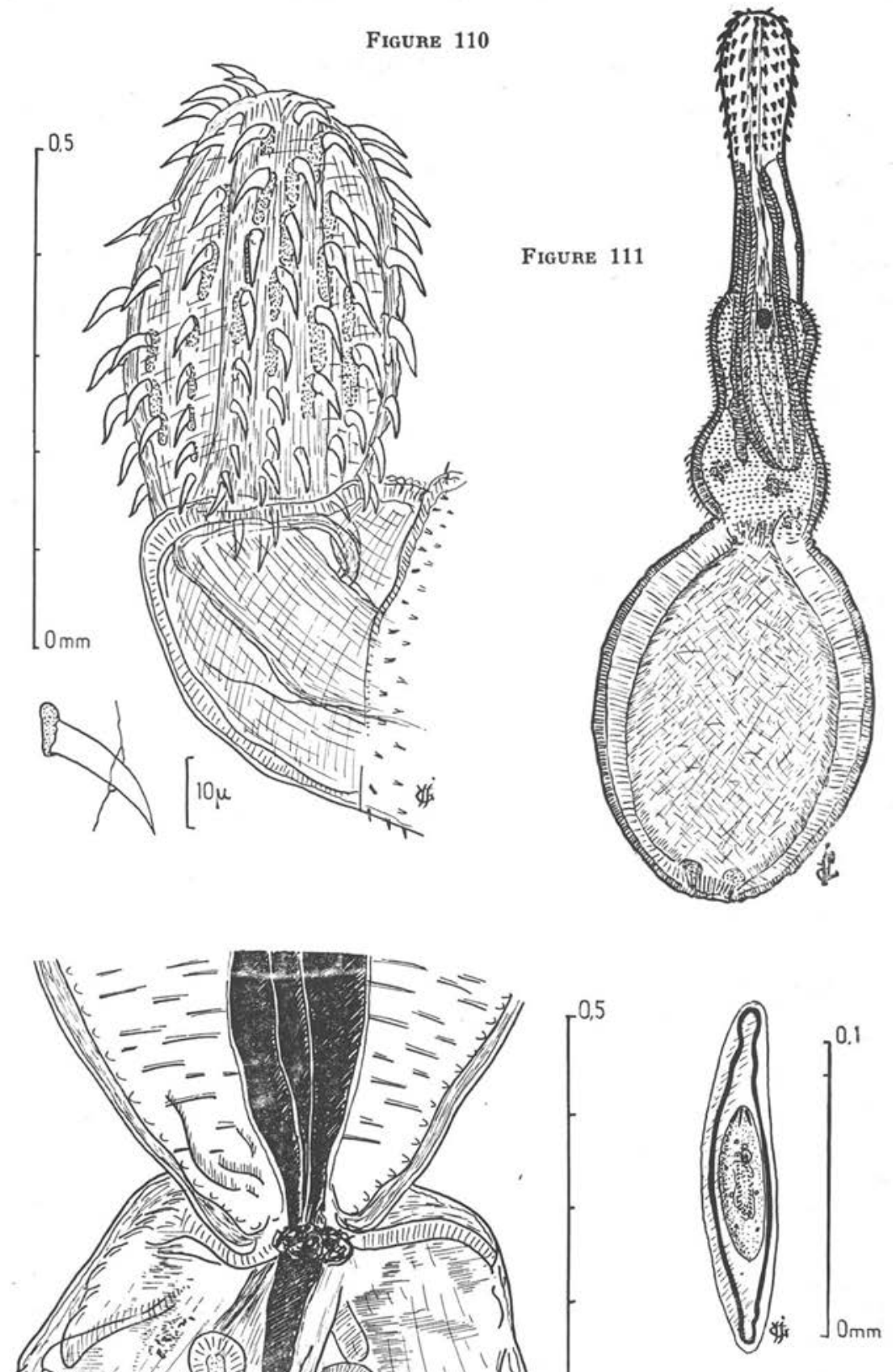

Figure 114 


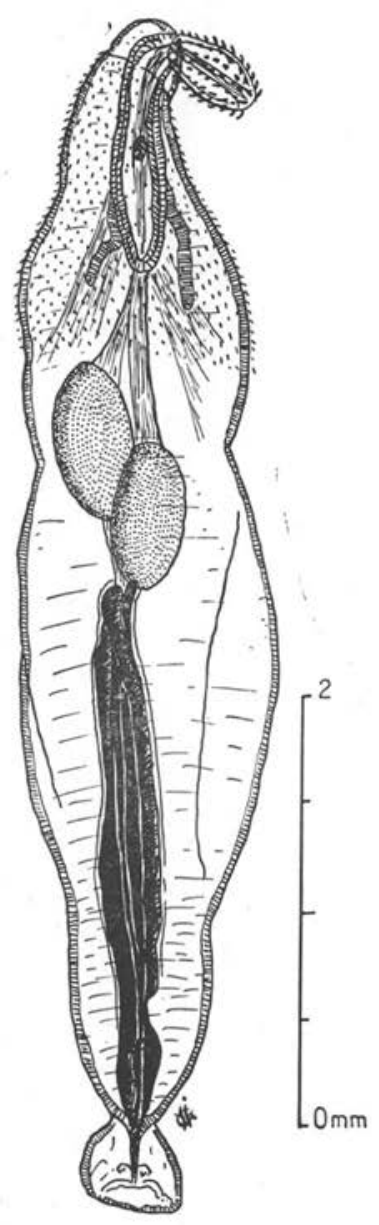

Figure 112

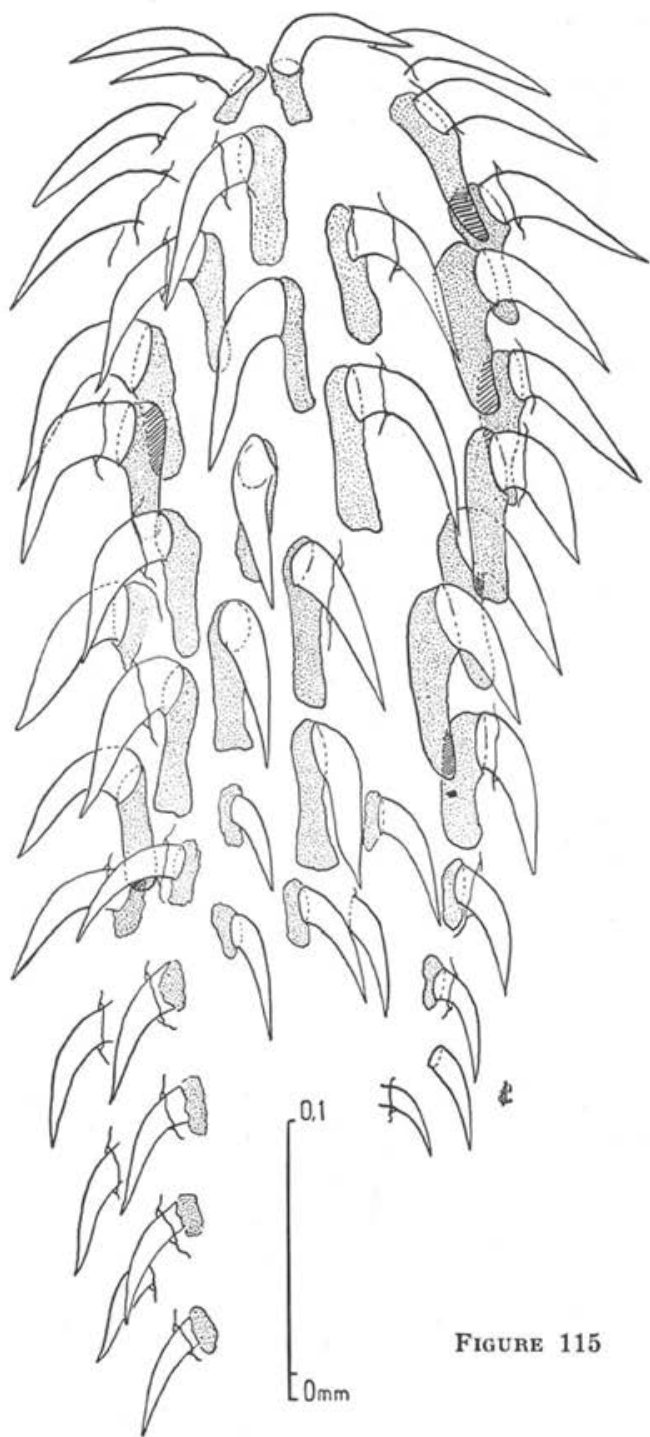

Fig. 110. - Polymorphus minutus (Zeder 1800), de l'intestin d'Anas platyrhynchos L. 1758. Proboscis et cou, Richelieu (I.-et-L.). Y. Golvan delin.

Fig. 111. - Polymmorphus minutus (Zeder 1800), juvénile de la cavité du corps de Gammarus pulex (L. 1758), Richelieu (I.-et-L.). Y. Golvan delin.

Fig. 112-115. - Polymorphus minutus (Zeder 1800) de l'intestin de Gallinula chloropus (L. 1758), Richelieu (I.-et-L.), 9-8-1952. Y. Golvan delin.

Fig. 112, figure d'ensemble d'un $\delta$; fig. 113, bourse copulatrice du même $\sigma^{*}$; fig. 114. œuf; fig. 115 , proboscis vu par la face ventrale, Yves Golvan delin. 


\section{PALAEACANTHOCEPHALA A. Meyer 1931}

Polymorphidae A. Meyer 1931

Polymorphus minutus (Zeder 1800) (fig. 110)

(fig. 112-115)

Polymorphus minutus (Zeder 1800), juv.

Plagiorhynchus crassicollis

(A. Villot 1875)

Centrorhynchus buteonis (Schrank 1788) (fig. 106 et 108-109)

Centrorhynchus aluconis

(O. F. Müller 1780)

(fig. 107)

Centrorhynchus aluconis

(O. F. Müller 1780), juv.

Centrorhynchus merulae

(Gmelin 1791)

Centrorhynchus teres

(Westrumb 1821)

Centrorhynchus (Sphaerirostris) picae (Rudolphi 1819)

Prosthorhynchus cylindraceus (Goeze 1782) intestin

intestin

cavités

du corps

intestin

intestin

intestin

intestin

intestin

intestin

intestin

cavité générale

cavité générale

et péritoine

péritoine

intestin

intestin

intestin

intestin

intestin

intestin

intestin
Strix aluco L. 1758

Corvus corone L. 1758

Anas platyrhynchos L. 1758 fer.

Gallinula chloropus

(L. 1758)

Gammarus pulex (L. 1758)

Asellus aquaticus (L. 1758)

Oedicnemus oedicnemus (L. 1758)

Buteo buteo (L. 1758)

Strix aluco sylvaticus Shaw 1809

Athene noctua (Scopoli 1769)

Tyto alba (Scopoli 1769)

Buteo buteo (L. 1758)

Sorex araneus L. 1758

Eliomys quercinus

(L. 1766)

Vipera aspis (L. 1766)

Merula merula (L. 1758)

Pica pica (L. 1758)

Pica pica (L. 1758)

Sturnus vulgaris L. 1758

Turdus viscivorus L. 1758

Turdus musicus L. 1758 


\begin{tabular}{|c|c|c|}
\hline & intestin & $\begin{array}{ccc}\text { Turdus } & \text { ericetorum Turton } \\
1817 & \text { ssp. philomelos } \\
\text { Brehm } & 1831 & \end{array}$ \\
\hline & intestin & Turdus sp. \\
\hline & intestin & $\begin{array}{l}\text { Merula merula (L. 1758) } \\
\quad \text { (fig. 103-104) }\end{array}$ \\
\hline & intestin & Pica pica (L. 1758) \\
\hline & intestin & $\begin{array}{l}\text { Garrulus glandarius } \\
\quad \text { (L. 1758) }\end{array}$ \\
\hline & intestin & $\begin{array}{l}\text { Coloeus monedula turrium } \\
\text { (Brehm 1831) }\end{array}$ \\
\hline & intestin & Corvus corone L. 1758 \\
\hline & intestin & $\begin{array}{l}\text { Athene noctua (Scopoli } \\
\text { 1769) }\end{array}$ \\
\hline & intestin & Buteo buteo (L. 1758) \\
\hline & intestin & Meleagris gallopavo L. 1758 \\
\hline id., adulte immature & & Sylvia atricapilla (L. 1758) \\
\hline $\begin{array}{l}\text { Prosthorhynchus cylindra- } \\
\text { ceus (Goeze 1782), juv. }\end{array}$ & $\begin{array}{l}\text { mésentère } \\
\text { et intestin }\end{array}$ & $\begin{array}{l}\text { Erinaceus europaeus } \\
\quad \text { L. } 1758\end{array}$ \\
\hline & & $\begin{array}{l}\text { Sorex minutus L. } 1766 \\
\text { Talpa europaea } \text { L. } 1758\end{array}$ \\
\hline & $\begin{array}{l}\text { cavité générale } \\
\text { et péritoine }\end{array}$ & $\begin{array}{l}\text { Eliomys quercinus } \\
\quad \text { (L. 1766) }\end{array}$ \\
\hline & mésentère & $\begin{array}{l}\text { Oryctolagus cuniculus (L. } \\
1758) \text { ferus }\end{array}$ \\
\hline Prosthorhynchus sp. & intestin & $\begin{array}{l}\text { Troglodytes troglodytes } \\
\quad \text { (L. 1758) }\end{array}$ \\
\hline
\end{tabular}

Echinorhynchidae Th. Sp. Cobbold 1879

Acanthocephalus anthuris intestin

(F. Dujardin 1845)
Triturus cristatus (Laurenti 1768)

Triturus vulgaris (L. 1758)

Triturus helveticus (Razoumowsky 1789)

Pomphorhynchidae S. Yamaguti 1939

Pomphorhynchus laevis intestin (Zoega in O.F. Müller 1776)
Leuciscus cephalus

(L. 1758)

Phoxinus phoxinus

(L. 1758) 

id.
Tinca tinca (L. 1758)
id.
Esox lucius L. 1758

\section{HIRUDINEA}

\section{Glossosiphoniidæ}

Protoclepsis tessellata (O. cavité nasale, Gallinula chloropus

F. Müller 1774)

$$
\text { surface de l'œil, (L. 1758) (49) }
$$

bouche,

exceptionnelle-

ment estomac

Anas platyrhynchos L. 1758

Hemiclepsis marginata (O. libre

F. Müller 1774)

Herpobdellidae

Herpobdella octoculata (L. libre

1758)

\section{Ichthyobdellidae}

Piscicola geometra

(L. 1758)

surface du corps Cyprinus carpio L. 1758

\section{OLIGOCHAETA}

\section{Branchiobdellidae}

Branchiobdella parasita

surface des branchies
Astacus pallipes Lereboullet 1858

(J.F. P. Braun 1805) for-

ma astaci Aug. Odier

1828

\section{Chœtogastridœ}

Chaetogaster sp. (50)

Theodoxia fluviatilis (L.

1758) et Ancylastrum ou

Ancylus

Chaetogaster limnaei K. v. sur le manteau Limnaea limosa (L. 1758)

Baer 1827

Limnaea stagnalis (L. 1758)

(49) Cette sangsue se gorge du sang de son hôte. Le sang est lentement digéré. Chez un individu rćcolté le 9 août, et observé sur le vivant chaque jour, c'est seulement le 94 août que j'ai constaté la disparition de toute trace du sang de l'hôte. R.-Ph. D.

(50) Environ 7 paires de bouquets de soies se composant (sauf les antérieurs et les postérieurs) de 9 à 11 soies terminées par deux crochets. 


\section{ARTHROPODES}

\section{LINGUATULIDES}
Linguatula serrata Froe- intestin Oryctolagus cuniculus lich 1789
(L. 1758) ferus

\section{CRUSTACES}

Copepoda (incl. Branchiura), parasites de Poissons

Lernaea esocina (Burmeis- surface du corps Phoxinus phoxinus ter 1833)

Argulus sp. surface du corps Tinca tinca (L. 1758) Abramis brama (L. 1758)

Leuciscus (Gardonus) rutilus (L. 1758)

Leuciscus cephalus (L. 1758)

Esox lucius L. 1758

Perca fluviatilis L. 1758

Des Copépodes cyclopoïdes ont été observés sur les branchies d'Anguilla anguilla (L. 1758)

\section{INSECTES}

\section{MALLOPHAGES}

AMBLYCERA

\section{Menoponida}

Amyrsidea perdicis (Denny 1842) Perdix perdix (L. 1758)

Colpocephalum flavescens Nitzsch Buteo buteo (L. 1758) 1818

Dennyus hirundinis (L. 1761) Apus apus (L. 1758)

Hohorstiella gigantea (Denny 1842) Streptopelia turtur (L. 1758)

Kurodaia subpachygaster (Piaget Tyto alba (Scopoli 1769) 1880)

Kurodaia longipes (Giebel 1874)

Carine (Athene) noctua (Scopoli 1769)

Kurodaia fulvofasciata (Piaget 1880) Buteo buteo (L. 1758)

Menacanthus fertilis (Nitzsch 1866) $\quad$ Upupa epops L. 1758 
Menacanthus spiniferus (Piaget 1885) Garrulus glandarius (L. 1758)

Menacanthus spiniferus (Piaget 1885) $\quad$ Sturnus vulgaris L. 1758 (51)

Menacanthus spinosus (Piaget 1880) Garrulus glandarius (L. 1758)

Menopon pallens Th. Clay $1949=$ Caccabis rufa (L. 1758)

$M$. pallescens Nitzsch 1874 non Giebel 1866

Perdix perdix (L. 1758)

Myrsidea anathorax (Nitzsch 1866) Pica pica (L. 1758)

Corvus corone L. 1758

Corvus frugilegus L. 1758

Myrsidea picae (L. 1758)

Pica pica (L. 1758)

Pseudomenopon tridens (Nitzsch in

Gallinula chloropus (L. 1758)

Burmeister 1838) = Ps. pilosum (Scopoli)

Podiceps ruficollis Pallas 1764

\section{Lœmobothriidœe}

Laemobothrium tinnunculi

(L. 1758)

Falco subbuteo L. 1758

Circus cyaneus (L. 1766)

Laemobothrium circi(Geoffroy 1762) Circus cyaneus (L. 1766)

\section{Ricinidae}

Ricinus fringillae De Geer $1778 \quad$ Fringilla coelebs L. 1758

\section{Trimenoponidae}

Trimenopon jenningsi (Kellog \& Cavia porcellus (L. 1758) Paine 1910)

\section{Gyropidœ}

Gliricola porcelli (Schrank 1781) Cavia porcellus (L. 1758)

Gyropus ovalis Nitzsch in Burmeis1838)

Cavia porcellus (L. 1758)

\section{ISCHNOCERA}

\section{Trichodectidae}

Trichodectes mustelae (Schrank Mustela erminea L. 1758 1803) 


\section{Philopteridae}

Acidoproctus stenopyx (Burmeister Mergus merganser L. 1758 (52) 1838)

Anaticola mergiserrati (De Geer Mergus merganser L. 1758 (53) 1778)

Nantoecus icterodes (Nitzsch 1818) Mergus merganser L. 1758 (53)

Ardeicola stellaris (Denny 1842) Botaurus stellaris (L. 1758) (52)

Brüelia glandarii (Denny 1842) Garrulus glandarius (L. 1758)

Brüelia gracilis (Nitzsch in Burmeis- Delichon urbica (L. 1758) ter 1838)

Brüelia varia (Nitzsch in Burmeister Corvus frugilegus L. 1758 1838)

Brüelia iliaci (Denny 1842)

Merula merula (L. 1758)

Brüelia marginalis (Nitzsch in Bur- Merula merula (L. 1758) meister 1838)

Coloceras damicorne (Nitzsch 1866) Streptopelia turtur (L. 1758)

Caccabis rufa (L. 1758)

Columba palumbus L. 1758

Columbicola columbae (L. 1758) Columba livia Gmelin 1789

Columba palumbus L. 1758

Streptopelia turtur (L. 1758)

Corvus frugilegus L. 1758

Craspedorrhynchus nisi (Denny Accipiter nisus (L. 1758) (1842)

Buteo buteo (L. 1758)

Craspedorrhynchus platystomus

(Nitzsch in Burmeister 1838)

Buteo buteo (L. 1758)

Pernis apivorus (L. 1758)

Accipiter gentilis (L. 1758)

Ardea purpurea L. 1766 (54)

Cuclotogaster heterogrammicus

Perdix perdix (L. 1758)

(Nitzsch 1866)

Degeeriella colymbina (Scopoli 1763)

Degeeriella giebeli Hopkins $1947=$ Buteo buteo (L. 1758)

D. fusca (Nitzsch 1861) (non Den-

ny 1842 )

(52) Etang de Gabian, Brenne (Indre).

(53) Etang du Renard, Mézières-en-Brenne (Indre).

(54) Hôte accidentel : spécimen déserteur. 
Docophorus communis (Nitzsch 1818)

Goniodes dissimilis Denny 1842

Goniodes colchici Denny 1842

Goniodes flaviceps Rudow 1869

Goniocotes bidentatus (Scopoli 1763)

Lipeurus caponis (L. 1758)

Philopterus corvi (L. 1758)

Philopterus subflavescens (Geoffroy 1762)

Quadraceps punctatus (Burmeister Larus ridibundus L. 1766 (56) 1838)

Rallicola cuspidatus (Scopoli 1763) Gallinula chloropus (L. 1758)

Saemundssonia lari (O. Fabricius Rissa tridactyla (L. 1758) 1780)

Strigiphilus ceblebrachys (Denny Buteo buteo (L. 1758) 1842)

Strigiphilus cursor (Burmeister 1838) Strix aluco sylvatica Shaw 1809

Strigiphilus rostratus (Burmeister Picus (Gecinus) viridis L. 1758 (57) 1838)

Strigiphilus cursitans (Nitzsch 1861) Carine (Athene) noctua (Scopoli 1769)

\section{ANOPLOURES}

\section{Hœmatopinidae}

Subfam. Linognathinae

Enderleinellus nitzschi Fahrenholz Sciurus vulgaris L. 1758

$1915=$ Enderleinellus sphaerocephalus (Nitzsch 1818)

Polyplax affinis (Burmeister 1839) Arvicola sapidus tenebricus G. Miller 1908

(55) La Celle-Guénaud (Indre-et-Loire).

(56) L'hôte des spécimens communiqués à Eugène Séguy avait été, par erreur, étiqucté «Larus melanocephalus Temminck 1820 ; c'est pourquoi, dans la « Faune de France », on trouve cet hôte mentionné par E. Séguy (1944, p. 299) au lieu de L. ridibundus L.

(57) Hôte accidentel : déserteur. 
Polyplax spinulosa (Burmeister 1839)

Polyplax reclinata (Nitzsch 1864)

Polyplax spiniger (Burmeister 1839)
Apodemus sylvaticus (L. 1758)

Sorex araneus L. 1758

Crocidura russula (Hermann in Zimmermann 1780) (58)

Arvicola amphibius (L. 1758) (58)

Arvicola sapidus tenebricus G. Miller 1908

id.

\title{
SIPHONAPTERES
}

\section{Pulicidae}

Archaeopsyllinae

Ctenocephalides canis (J. Curtis Vulpes vulpes (L. 1758) 1826)

Ctenocephalides felis felis (Bouché Felis catus L. 1758, domest. 1835)

Archaeopsylla erinacei erinacei

(Bouché 1835)

Vulpes vulpes (L. 1758)

Erinaceus europaeus L. 1758

\section{Spilopsyllinae}

Spilopsyllus cuniculi (C. W. Dale Martes foina (Erxleben 1777)

1878)

\author{
Vulpes vulpes (L. 1758) \\ Oryctolagus cuniculus (L. 1758) ferus \\ Sciurus vulgaris L. 1758
}

Ischnopsyllidae Ischnopsyllinae

Rhinolophopsylla unipectinata uni- Rhinolophus hipposideros (Bechstein pectinata (Taschenberg 1880) 1800)

Hystrichopsyllidae Hystrichopsyllinae

Hystrichopsylla talpae (J. Curtis Talpa europaea L. 1758 1826)

Clethrionomys glareolus (Schreber 1792)

Typhloceras poppei Wagner 1903 Apodemus sylvaticus (L. 1758)

(58) Hôte accidentel. 
Leptopsyllinae

Leptopsylla segnis (Schönherr 1816) Rattus rattus (L. 1758)

Mus musculus L. 1758

Palaeopsylla minor minor (Dale Talpa europaea L. 1758 1878)

\section{Neopsyllinae}

Ctenophthalmus agyrtes (Heller Apodemus sylvaticus (L. 1758) 1896)

Ctenophthalmus nobilis vulgaris F. G. A. M. Smit

Ctenophthalmus congener congener N. C. Rothschild 1907

Doratopsylla dasycnemus dasycnemus (N. C. Rothschild 1897)

Rhadinopsylla pentacanthus (Rothschild 1897)

\section{Dolichopsyllidae}

\section{Dolichopsyllinae}

Monopsyllus sciurorum (Schrank Sciurus vulgaris L. 1758 1803)

Nosopsyllus fasciatus (L.

A. G. Bosc d'Antic 1801) 1792)

Sorex araneus L. 1758 mermann 1780)

Talpa europaea L. 1758

Arvicola terrestris (L. 1758) Miller 1908

Mus musculus L. 1758

Apodemus sylvaticus (L. 1758)

Arvicola terrestris (L. 1758) 1792)

Eliomys quercinus (L. 1766)

Crocidura russula (Her-
Clethrionomys glareolus (Schreber

Crocidura russula (Hermann in Zim-

Arvicola sapidus tenebricus Gerrit

Crocidura russula (Hermann in Zimmermann 1780)

Clethrionomys glareolus (Schreber

Martes foina (Erxleben 1777) mann in Zimmermann 1780)

Clethrionomys glareolus

(Schreber 1792)

Nosopsyllus londiniensis (Rothschild Mus musculus L. 1758 1903)

Paraceras melis (J. Curtis 1832) Vulpes vulpes (L. 1758)

Ann. de Parasitologie, T. XXXVI, N $3,1961$. 


\section{DIPTERES}

Sarcophagidae parasites d'ACRIDIENS

Sarcophaga exuberans larve dans

Pandellé 1826

Sarcophaga haematodes

Meigen 1826

Sarcophagidae non identi- larves dans fiés

larve dans

\author{
Euchorthippus pulvinatus \\ (Fischer de Waldheim \\ 1846) \\ Gomphocerus rufus \\ (L. 1758) \\ Edipoda caerulescens (L. \\ 1758) \\ Stauroderus bicolor (Char- \\ pentier 1825) \\ Stauroderus biguttatus (L. \\ 1758)
}

\section{Tachinida parasites d'ACRIDIENS}

Acemyia acuticornis (Mei- larves dans gen 1824)
Chorthippus dorsatus (Zetterstedt 1821)

Euchorthippus pulvinatus (Fischer de Waldheim 1846)

Pezotettix giornai (Rossi 1794)

Stauroderus bicolor (Charpentier 1825)

\section{Phasiinae parasites d'HETEROPTERES par Claude DUPUIS}

Subtrib. Leucostomatina

Dionaea forcipata (Meigen 1824) Enoplops scapha (Fabricius 1794)

Leucostoma analis (Meigen 1824) Rhopalus subrufus (Gmelin 1788) Dupuis 1953

Enoplops scapha (Fabricius 1794)

Leucostoma meridiana (Rondani Myrmus miriformis (Fallén 1807) 1868) 
Stictopleurus punctatonervosus (Goeze 1778)

Leucostoma sp.? [? meridiana (Ron- Corizus hyoscyami (L. 1758) dani 1868)]

Leucostomatina sp.?

Coriomeris hirticornis (Fabricius 1794)

Syromastus rhombeus (L. 1767) (= Verlusia rhombea auct.)

Subtrib. Cylindromyiina

Neocyptera auriceps (Meigen 1838) Aelia acuminata (L. 1758)

Eurydema oleracea (L. 1758)

Chaetocyptera bicolor (Olivier 1811) Rhaphigaster nebulosa (Poda 1761)

Cylindromyia brevicornis (Loew Dolycoris baccarum (L. 1758) 1844)

Cylindromyia pilipes (Loew 1844) Holcostethus vernalis (Wolff 1804)

Cylindromyia brassicaria (Fabricius Dolycoris baccarum (L. 1758) 1775)

Subtrib. Phaniina

Phania vittata Meigen 1824

Arma custos (Fabricius 1794)

Subtrib. Allophorin 7

Hyalomyia pusilla (Meigen 1824) Cymus glandicolor (Hahn 1832)

Kleidocerus ericae (Horvâth 1910)

Nysius jacobeae (Schilling 1829)

Nysius lineatus (Costa 1852)

Brumptallophora aurigera (Egger Gonocerus juniperi Herrich-Schäffer 1860) 1839

Palomena prasina (L. 1761)

Rhaphigaster nebulosa (Poda 1761)

Allophora hemiptera (Fabricius Tropicoris rufipes (L. 1758)

1794)

Subtrib. Helomyiina

Helomyia lateralis (Meigen 1824) Alydus calcaratus (L. 1758)

Aelia acuminata (L. 1758)

Carpocoris pudicus (Poda 1761)

Dolycoris baccarum (L. 1758)

Graphosoma italicum (Müller 1766) 
Eurygaster maura (L. 1758) China 1927

Subtrib. Ectophasiina

Chryseria helluo (Fabricius 1805) Eurygaster testudinaria (Geoffroy in Fourcroy 1785) China 1927

Clytiomyia continua (Panzer 1798) Eurydema oleracea (L. 1758)

Eurydema ornata (L. 1758) Stichel 1926

Clytiophasia dalmatica (Robineau- Graphosoma italicum (Müller 1766) Desvoidy 1830)

Ectophasia rostrata (Egger 1860)

Lygaeus saxatilis (Scopoli 1763)

Coreus marginatus (L. 1758)

Gonocerus acuteangulatus (Goeze 1778)

Piezodorus lituratus (Fabricius 1794)

Aelia acuminata (L. 1758)

Carpocoris pudicus (Poda 1761)

Dolycoris baccarum (L. 1758)

Holcostethus vernalis (Wolff 1804)

Eurydema oleracea (L. 1758)

Eurydema ornata (L. 1758) Stichel 1926

Graphosoma italicum (Müller 1766)

Eurygaster austriaca (Schrank 1778)

Eurygaster maura (L. 1758) China 1927

Ectophasia rubra (Girschner 1888) Coranus aegyptius (Fabricius 1775)

Lygaeus equestris (L. 1758)

Lygaeus saxatilis (Scopoli 1763)

Alydus calcaratus (L. 1758)

Camptopus lateralis (Germar 1817)

Corizus hyoscyami (L. 1758)

Coreus marginatus (L. 1758)

Gonocerus acuteangulatus (Goeze 1778)

Gonocerus juniperi Herrich-Schäffer 1839 
Syromastus rhombeus (L. 1767) (= Verlusia rhombea auct.)

Aelia acuminata (L. 1758)

Carpocoris pudicus (Poda 1761)

Dolycoris baccarum (L. 1758)

Eurydema ornata (L. 1758) Stichel 1926

Eurydema ventralis Kolenati 1846

Graphosoma italicum (Müller 1766)

Eurygaster austriaca (Schrank 1778)

Eurygaster maura (L. 1758) China 1927

Eurygaster testudinaria (Geoffroy in Fourcroy 1785) China 1927

Subtrib. Gymnosomatina

Gymnosoma clavata (Rohdendorf Piezodorus lituratus (Fabricius 1794) 1947)

Carpocoris pudicus (Poda 1761)

Dolycoris baccarum (L. 1758)

Eurydema ornata (L. 1758) Stichel 1926

Graphosoma italicum (Müller 1766)

Gymnosoma rotundata (L. 1758)

Palomena prasina (L. 1761)

Gymnosoma dolycoridis Dupuis 1960 Piezodorus lituratus (Fabricius 1794)

Carpocoris pudicus (Poda 1761)

Dolycoris baccarum (L. 1758)

Holcostethus vernalis (Wolff 1804)

Graphosoma italicum (Müller 1766)

Gymnosoma carpocoridis Dupuis Piezodorus lituratus (Fabricius 1794) 1961

Carpocoris pudicus (Poda 1761)

Dolycoris baccarum (L. 1758)

Holcostethus vernalis (Wolff 1804)

Graphosoma italicum (Müller 1766)

Cystogaster globosa (Fabricius 1775) Aelia acuminata (L. 1758)

Neotiglossa leporina (Herrich-Schäf-

fer 1830) 


\section{Nycteribiidce}

Nycteribia (Celeripes) biarti-

Plecotus auretus (L. 1758)

culata Hermann

et autres Nycteribiidae sur

divers Chiroptères

Hippoboscidae Parasites d'OISEAUX

Ornithomyia avicularia (L. sur Garrulus glandarius (L. 1758) 1761)

Stenopteryx hirundinis (L. dans Hirundo (Chelidon) rustica L. 1761) le nid 1758

Ceratopogonidae

Culicoides chiopterus Meigen (59)

agressif pour l'homme

Culicoides heliophilus Edwards

Psychodidae

Phlebotomus perniciosus

Newstead 1911

agressif pour l'hommo

\section{Culicidae}

Anopheles maculipennis maculipennis Meigen 1818

Anopheles maculipennis messeae Falleroni 1926

Anopheles maculipennis melanoon L. W. Hackett 1934

Anopheles labranchiae atroparvus P. H. van Thiel 1927

Anopheles claviger (Meigen 1804)

Anopheles plumbeus Haliday et Stephens 1828

Theobaldia annulata (Schrank 1776)

Theobaldia longeareolata (Macquart 1838)

Theobaldia fumipennis (Stephens 1825)

Theobaldia morsitans (Theobaldi 1901)

Culex pipiens pipiens L. 1758, variété eurygame, anautogène et ornithophile et variété sténogame, anautogène et aussi ornithophile

Culex modestus Ficalbi 1889

Culex hortensis Ficalbi 1889

Cùlex territans Walker 1856

Aèdes cinereus Meigen 1818

Aedes vexans (Meigen 1830)

Aedes sticticus Meigen 1838

Aedes rusticus (Rossi 1790)

(59) $\mathrm{D}^{r}$ Hervẻ Harant determin. 
Aedes punctor (Kirby 1837)

Aedes communis (De Geer 1776)

Aedes cantans (Meigen 1818)

Aedes annulipes (Meigen 1830)

Aedes geniculatus (Olivier 1791)

Aedes excrucians Walker 1856

Orthopodomyia pulchripalpis (Rondani 1872)

Taeniorhynchus richiardii (Ficalbi 1889)

\section{Tabanidae}

Tabanus bromius L. 1758

Therioplectes gigas (Herbst 1787)

Therioplectes nemoralis (Meigen 1820)

Therioplectes quatuornotatus (Meigen 1820)

Haematopota pluvialis (L. 1758)

Chrysops caecutiens (L. 1758)

Chrysops relictus Meigen 1820

\section{ACARIENS}

A) de MAMMIFERES (excl. Ixodidae)

\section{Trombiculidae}

Trombicula autumnalis (Shaw larve sur Mustela nivalis L. 1766 1790)

Trombicula sp.

Euschöngastia sp.

larve sur Oryctolagus cuniculus L., fer.

larve dans Mustela nivalis L. 1766 les fosses nasales

Dasyponissidae Fonseca 1940

Dermanyssus Kolenati 1859

Dermanyssus sp.

sur

Myotis emarginatus (E. Geoffroy-St-Hilaire 1806)

\section{Listrophoridae}

Listrophorus mustelae Mé- sur Mustela nivalis L. 1766 gnin 1885

Listrophorus leuckarti Pagenstecher 1861

sur 4rvicola sapidus tenebricus Gerrit Miller 1908

B) d'OISEAUX

Dasyponissidae Fonseca 1940

Dermanyssus Kolenati 1859 
Dermanyssus gallinae (De Geer Gallus gallus (L. 1758), domest. 1778)

Dermanyssus sp.

Turdus sp. juv.

Trombiculidae Ewing 1944

Trombiculinae Ewing 1929

Trombicula autumnalis (Shaw 1790) Turdus merula L. 1758 (larve)

Sarcoptidae Mégnin 1880

Cnemidocoptes mutans Robin et Lan- Gallus gallus (L. 1758), domest. quetin 1859

Pterolichidae Trouessart 1915

Gabucinia (= Pterolichus) delibata Corvus corone L. 1758 (Robin 1877)

Pteronyssus brevipes Berlese 1884

Corvus frugilegus L. 1758

Dryobates medius (L. 1758)

Pteronyssus picinus (C. L. Koch 1840)

Pteronyssus sp.

Dryobates medius (L. 1758)

Dryobates medius (L. 1758)

Pteronyssus monoplax Jean Gaud = Pt. quadratus Halles 1882 pro parte

Dryobates medius (L. 1758)

Dryobates major (L. 1758)

Pteronyssoides truncatus (Trouessart Sturnus vulgaris L. 1758 1885)

Kramerella lunulata (Halles 1878)

Carine (= Athene) noctua (Scopoli 1769)

Analgesidae Trouessart 1915

Analges corvinus Robin et Mégnin 1877

Analges spiniger Giebel 1871

Analges cf. unidentatus Berlese 1883

Megniniella gallinulae (Buchholz 1869)

Megniniella fulicae Jean Gaud 1958 $=$ Megninia rallorum Trouessart 1885 pro parte

Megniniella sp.

Gallinula chloropus (L. 1758)

Mesalges pici-majoris (Buchholz Dryobates medius (L. 1758) 1870)

Mesalges oscinum (Koch 1840)

Dryobates major (L. 1758)

Podiceps ruficollis (Pallas 1764)

Colynisus sp.

Podiceps ruficollis (Pallas 1764) 


\section{Proctophyllodidae}

Proctophyllodes sylviae Jean Gaud Sylvia atricapilla (L. 1758) 1957

Aegithalos caudatus (L. 1758)

Proctophyllodes pinnatus (Nitzsch Emberiza cirlus L. 1766 1818)

Proctophyllodes sp.

Sitta europaea (L. 1758)

Proctophyllodes dollfusi Jean Gaud Sitta europaea (L. 1758)

Proctophyllodes rubeculinus Koch Erythacus rubecula (L. 1758) 1840

Proctophyllodes musicus Vitzthum Turdus merula L. 1758 1922

Pterodectes cylindricus (Robin \& Pica pica (L. 1758) Mégnin 1868)

Pterodectes sp. Corvus corone L. 1758

Trouessartia simillima J. Gaud Erythacus rubecula (L. 1758) 1957

Trouessartia rosteri (Berlese 1883) Sturnus vulgaris L. 1758

Falculiferidœe Oudemans 1908

Falculifer rostratus (Buchholz 1869) Columba palumbus L. 1758

Columba oenas L. 1758

C) des fosses nasales d'OISEAUX

Dasyponyssidae Fonseca 1940

Rhinonyssinae Trouessart 1895

Rallinyssus strandtmanni Simon

Gretillat 1961

Gallinula chloropus (L. 1758)

\section{Ixodidés (incl. Argasidés)}

Dans cette liste, sont mentionnées des récoltes à Richelieu $(\mathrm{R})$ et dans d'autres localités du département d'Indre-et-Loire, ainsi que dans quelques départements voisins $(60)$.

Ixodidae Murray 1877

Ixodinae Vitzthum 1941

Ixodes ricinus (L. 1758)

adulte sur Homo sapiens L. 1758 (R)

Canis familiaris L. 1758 (R)

(2) (3)

(60) Renseignements communiqués par le Dr P.-C. Morel.

Les chiffres entre parenthèses renvoient aux localités numérotées 1 a 13 , mentionnées au bas des pages 339-340. 
Martes foina (Erxleben 1777) (R)

Cervus elaphus L. 1758 (1) (2) (6)

Lepus europaeus Pallas 1778 (4) (5)

Sciurus vulgaris L. 1758 (R)

nymphe sur Homo sapiens L. 1758 (R)

Cervus elaphus L. 1758 (2)

Sciurus vulgaris L. 1758 (R)

Erinaceus europaeus L. 1758 (R)

Falco tinnunculus L. 1758 (R)

Lacerta viridis (Laurenti 1768) (R)

larve sur Cervus elaphus L. 1758 (2)

Apodemus sylvaticus (L. 1758) (R)

Sciurus vulgaris L. 1758 (R)

Oryctolagus cuniculus (L. 1758) ferus (R)

Turdus ericetorum Turton 1807 (R)

Turdus merula L. 1758 (R)

Lacerta viridis (Laurenti 1768) (R)

llxodes sp. (? ricinus L.) larve sur Clethrionomys glareolus (Schreber 1792)

Ixodes hexagonus Leach 1815 adulte sur Mustela nivalis L. 1766 (R)

Mustela putorius L. 1758 (R)

Erinaceus europaeus L. 1758 (R)

Canis familiaris L. 1758 (2)

nymphe sur Mustela nivalis L. 1766 (R)

Felis catus L. 1758 domest. (R)

Canis familiaris L. 1758 (2)

larve sur Mustela nivalis L. 1766 (R) 
Ixodes autumnalis Leach 1815 $=I$. canisuga $\mathrm{G}$. Johnston 1849

Ixodes lividus Koch 1844

Ixodes frontalis (Panzer 1798)

Ixodes passericola Schulze 1933

Eschatocephalus vespertilionis (Koch 1844) adulte sur

Canis familiaris L. 1758 (2)

Vulpes vulpes (L. 1758) (8)

nymphe Riparia riparia (L. 1758) (7) dans le nid

adulte sur Turdus merula L. 1758 (R)

nymphe et Sturnus vulgaris L. 1758 (R) larve sur

nymphe sur Pipistrellus pipistrellus (Schreber 1775) (9)

\section{Rhipicephalinae Vitzthum 1941}

Hyalomma anatolicum Koch nymphe sur Anthus trivialis (L. 1758) (R) 1844

Hyalomma detritum scapense adulte sur Equus caballus L. 1758 (10) Schulze 1918

Haemalastorinae Oudemans 1936

Haemaphysalis punctata Ca- adulte sur Cervus elaphus L. 1758 (2) nestrini \& Fanzago 1877

Haemaphysalis (Alloceras) iner- adulte Cervus elaphus L. 1758 (2) mis Birula 1896

Haemaphysalis concinna Koch nymphe sur Cervus elaphus L. 1758 (1) (2) 1844

Canis familiaris L. 1758 (2)

Dermacentor marginatus (Sulzer 1776)

Dermacentor pictus (Hermann adulte sur Hôtes inconnus (ou libres?) (11) (12) 1804)

Canis familiaris L. 1758 (5) (9) (13) (14) (16) (17)

Cervus elaphus L. 1758 (2) (6)

(1) Amboise (Indre-et-Loire).

(2) Lancosme (Indre).

(3) La Ferté-Vidame (Indre-et-Loire).

(4) Romorantin (Loir-et-Cher).

(5) Poitiers (Vienne).

(6) Chitré, près Verneuil-sur-Vienne (Haute-Vienne).

(7) Argenton (Creuse).

(8) St-Christophe (Indre-et-Loire).

(9) Tours (Indre-et-Loire).

(10) Sillé-le-Guillaume (Sarthe).

(11) Savonnières, près Tours (Indre-et-Loire).

(12) Châtellerault (Vienne).

(13) Chinon (Indre-et-Loire). 
Hôtes inconnus (ou libres?)

(15) (18) (R)

Dermacentor reticulatus (J. C. adulte

Fabricius 1794) libre

\section{Argasidae G. Canestrini 1890}

Carios vespertilionis P.-A. La- larve sur Eptesicus serotinus (Schreber treille 1796 1775) (R)

ACARIENS parasites ou commensaux d'INSECTES et MYRIAPODES

\section{Parasitiformes}

A. Gamasidés

Macrochelida

Macrocheles sp.

Cophrolaspis glaber (J. Müller 1859)

Pœcilochirida

Poecilochirus necrophori Vitzthum 1930

Poecilochirus subterraneus (J. Müller 1859)

Gamaside indéterminé

Gamaside indéterminé

\section{Laelaptidae}

Lasioseius sp.

B. Uropodina

\section{Uropodidae}

Pseudouropoda vegetans (De Geer 1778)

(14) Loches (Indre-et-Loire).

(15) La Flèche (Sarthe).

(16) Sous-Biard (Vienne).

(17) Lathus (Vienne).

(18) Saumur (Maine-et-Loire).
Necrophorus humator Goeze 1777

Necrophorus vespillo L. 1758

Geotrupes vernalis (L. 1758)

Necrophorus humator Goeze 1777

Necrophorus vestigator Herschel 1807

Necrophorus humator Goeze 1777

Bombus sp.

Necrophorus fossor (L. 1758)

Necrophorus vespillo L. 1758

Dorcus parallelipipedus (L. 1767) 
C. Trachytina

\section{Trachytidae}

Neoseius novus (Oudemans 1902)

Polyaspida

Polyaspis patavinus Berlese 1881
Necrophorus vespillo L. 1758

Necrophorus vespilloides

Herbst 1784

Lucanus cervus L. 1767

Dorcus parallelipipedus 1767)

Dorcus parallelipipedus (L. 1767)

Lucanus cervus L. 1767

\section{Thrombidiiformes}

Thrombidiidae indét. sur Panorpa communis L. 1758

Lebertiidae

Lebertia sp., larves, sur Orthocladius sp.

\section{Sarcoptiformes}

\section{Tyroglyphidae}

Histiostoma sp.

Histiostoma sp.

\section{Caloglyphidae}

Caloglyphus geotruporum Zachv. 1941

Caloglyphus sp.

\section{Canestriniida}

Canestrinia dorcicola Berlese 1881

Paramansia menthastri Cooreman 1950

Percanestrinia saetolata Cooreman 1950

Pseudamansia chrysomelinus (C. L. Koch 1841)

\section{Linobiidae}

Linobia coccinellae (Scopoli 1763)
Oeceoptoma thoracicum (L. 1758)

Paederus ruficollis F. 1787

Polydesmus complanatus (L. 1767)

Geotrupes stercorosus (Scriba 1791) (sous les élytres)

Polydesmus complanatus (L. 1767)

Dorcus parallelipipedus (L. 1767)

Chrysolina menthastri Suffrian 1850

Procrustes purpurascens (Fabricius 1787)

Timarcha goettingensis (L. 1758)

Chrysomela populi L. 1758 